\title{
Artificial Cognition in Production Systems
}

Alexander Bannat, Thibault Bautze, Michael Beetz, Juergen Blume, Klaus Diepold, Christoph Ertelt, Florian Geiger, Thomas Gmeiner, Tobias Gyger, Alois Knoll, Christian Lau, Claus Lenz, Martin Ostgathe, Gunther Reinhart, Wolfgang Roesel, Thomas Ruehr, Anna Schuboe, Kristina Shea, Ingo Stork genannt Wersborg, Sonja Stork, William Tekouo, Frank Wallhoff, Mathey Wiesbeck, and Michael F. Zaeh

\begin{abstract}
Today's manufacturing and assembly systems have to be flexible to adapt quickly to an increasing number and variety of products, and changing market volumes. To manage these dynamics, several production concepts (e.g., flexible, reconfigurable, changeable or autonomous manufacturing and assembly systems) were proposed and partly realized in the past years.

This paper presents the general principles of autonomy and the proposed concepts, methods and technologies to realize cognitive planning, cognitive control and cognitive operation of production systems. Starting with an introduction on the historical context of different paradigms of production (e.g., evolution of production and planning systems), different approaches for the design, planning, and operation of production systems are lined out and future trends towards fully autonomous components of an production system as well as autonomous parts and products are discussed. In flexible production systems with manual and automatic assembly tasks, human-robot cooperation is an opportunity for an ergonomic and economic manufacturing system especially for low lot sizes. The state-of-the-art and a cognitive approach in this area are outlined. Furthermore, introducing self-optimizing and self-learning control systems is a crucial factor for cognitive systems. This principles are demonstrated by a quality assurance and process control in laser welding that is used to perform improved quality monitoring. Finally, as the integration of human workers into the workflow of a production system is of the highest priority for an efficient production, worker guidance systems for manual assembly with environmentally- and situationally dependent triggered paths on state-based graphs are described in this paper.
\end{abstract}

Note to Practitioners-Today's manufacturing enterprises have to face a number of challenges in a turbulent environment that originates amongst other from saturated markets, unprecedented and abrupt changes in market demands, an ever increasing number of product variants and smaller lot sizes. A recent research trend in Germany is the so called Cognitive Factory, where artificial cognitive capabilities are introduced to the control of production systems. The applications range from production planning and control, human-robot-cooperation, automatic robot programming to intuitive worker guidance systems.

The concept of fully automated production systems is no longer a viable vision, as it has been shown, that the conventional automa-

Manuscript received December 10, 2009; accepted February 18, 2010. Date of publication July 26, 2010; date of current version January 07, 2011. This paper was recommended for publication by Associate Editor Y. Ma and Editor M. Zhou upon evaluation of the reviewers' comments. This ongoing work is supported by the DFG Excellence Initiative Research Cluster Cognition for Technical Systems-CoTeSys, see www.cotesys.org for further details.

A. Bannat, T. Bautze, M. Beetz, J. Blume, K. Diepold, C. Ertelt, F. Geiger, T. Gmeiner, T. Gyger, A. Knoll, C. Lau, C. Lenz, M. Ostgathe, G. Reinhart, W. Roesel, T. Ruehr, K. Shea, I. Stork genannt Wersborg, W. Tekouo, F. Wallhoff, M. Wiesbeck and F. M. F. Zaeh are with Technische Universitaet Muenchen, 80333 Munich, Germany (e-mail: wolfgang.roesel@iwb.tum.de).

A. Schuboe and S. Stork are with Ludwig-Maximilians-Universitaet Muenchen, 80539 Munich, Germany.

Color versions of one or more of the figures in this paper are available online at http://ieeexplore.ieee.org.

Digital Object Identifier 10.1109/TASE.2010.2053534 tion is not able to deal with the ever-rising complexity of modern production systems. Especially, a high reactivity, agility and adaptivity that is required by modern production systems, can only be reached by human operators with their immense cognitive capabilities, which enable them to react to unpredictable situations, to plan their further actions, to learn and to gain experience and to communicate with others. Thus, new concepts are required, that apply these cognitive principles to the planning processes and control systems of production systems.

Index Terms-Adaptive control, assembly systems, assistance systems, autonomous processes, cognitive factory, computer aided design, computer-aided manufacturing, computer aided process planning, computer integrated manufacturing, flexible manufacturing systems, human-robot cooperation, manufacturing systems, multi-agent systems, paradigms of production, production planning and control, quality assurance, radio-frequency identification, reconfigurable manufacturing systems.

\section{INTRODUCTION}

$\mathbf{T}$ ODAY's manufacturing enterprises have to face a number of challenges in a turbulent environment that originates amongst other from saturated markets, unprecedented and abrupt changes in market demands, an ever increasing number of product variants and smaller lot sizes [1], [2]. This trend is intensified by concepts such as mass customization and individualization, which promise the creation of unique products to satisfy the needs of nearly every customer. In addition, the globalization of the economy has intensified the worldwide competition and caused a continuous pressure on production costs [3]. Therefore, companies can only compete successfully if they offer products and services that meet the customer's individual requirements without sacrificing cost effectiveness or product quality [4], [5]. This results in large-scale fluctuations of demand, increasing product variants with specific configurations, random dispatching of orders, short delivery lead times of products and short product life cycles [6], [7].

Regarding the growing dynamics, the planning and control of production systems has become increasingly complex regarding flexibility and productivity as well as the decreasing predictability of processes [8]. In spite of the aforementioned general conditions, Koren [5] stated that every manufacturing enterprise and therefore every production system should pursue the following three main objectives:

- providing capability for rapid responsiveness;

- enhancement of product quality;

- production at low cost.

On the one hand, these requirements can be satisfied through highly stable and repeatable processes. On the other hand, these 


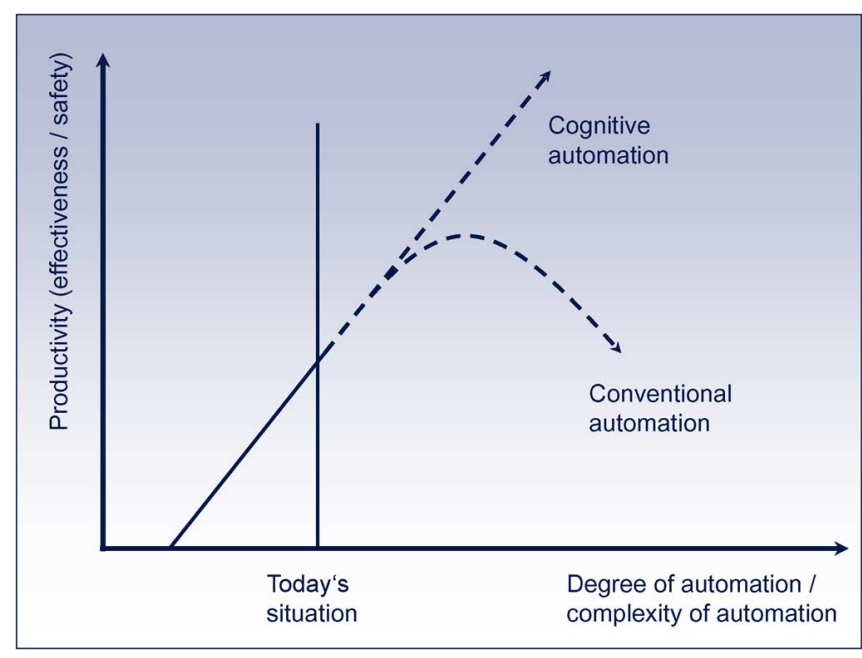

Fig. 1. Need for cognitive automation to improve productivity [12].

requirements can be achieved by creating short response times to deviations in the production system, the production process, or the configuration of the product in coherence to overall performance targets [9]. In order to obtain short response times, a high process transparency [9] and the reliable provisioning of the required information to the point of need at the correct time and without human intervention [10] is essential. However, the success of those adaptive and responsive production systems highly depends on real-time and operation-synchronous information from the production system, the production process and the individual product [2]. Recent information and communication technologies such as radio frequency identification (RFID), cameras, laser scanners, actual state information from programmable logic controllers (PLC) and robot controllers (RC) are essential elements to collect real-time data from a production facility and its subsystems [11]. Pertinent information from the shop floor can then be integrated continuously to production planning and control instances. The realization of this aspect can be considered as the essential element for the shift from offline planning systems to online and closed-loop control systems in production environments [4].

However, it can be stated, that the concept of fully automated production systems is no longer a viable vision, as it has been shown, that the conventional automation is not able to deal with the ever-rising complexity of modern production systems. Especially, a high reactivity, agility and adaptivity that is required by modern production systems, can only be reached by human operators with their immense cognitive capabilities, which enable them to react to unpredictable situations, to plan their further actions, to learn and to gain experience and to communicate with others. Thus, new concepts are required, that apply these cognitive principles to the planning processes and control systems of production systems (see Fig. 1).

In the following sections, the historical context and development of production paradigms in the last decades are described and the state-of-the-art as well as novel approaches are outlined. This paper hereby covers topics of the production planning and control, the planning of automated processes and operations, as well as of hybrid and manual workstations (see Fig. 2).
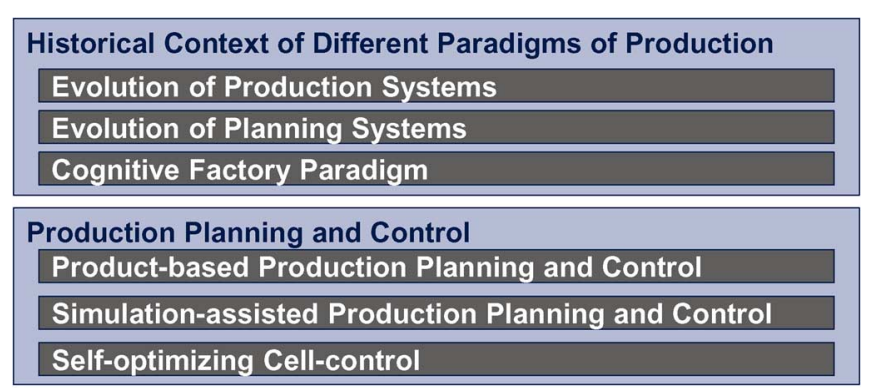

\begin{tabular}{|l|}
\hline Automated Processes and Operations \\
\hline Machining Planning \\
\hline Fixture Design and Reconfiguration \\
\hline Robot-guided Quality Inspection \\
\hline
\end{tabular}

Hybrid and Manual Processes and Operations

Human-robot Co-operation

Quality Assurance and Process Control in Laser Welding Assistance Systems

Fig. 2. Covered topics of this paper.

\section{Historical CONTEXT OF DifFerent PARADIGMS OF PRODUCTION}

\section{A. Evolution of Production Systems}

In recent decades, many researchers identified the necessity to cope with changing boundary conditions and to develop novel manufacturing paradigms [1], [13], [14]. Dedicated manufacturing lines with fixed tooling and automation were developed for mass production, which aims for the manufacturing of high quantities of identical parts at the required quality for low costs. As a consequence, the final costs per part have been significantly reduced [1], [14], [15].

The emerging diversifications of products lead to the development of new approaches for efficient planning and control of manufacturing systems. Examples include Computer Integrated Manufacturing (CIM) with the well-known Flexible Manufacturing Systems (FMS). These developments aim for the economic production of and change between predefined product variants of different volumes [14], [15]. FMS, however, are characterized by rigid hardware and software architectures with completely predetermined and manually defined production processes. This entails limited capabilities in terms of upgradeability, customization, changes in production capacity and reaction to unforeseen events (e.g., machine failure, variation in quality) [1], [4].

Traditional approaches, which consist mostly of rigid, hierarchical control architectures, have been unable to deal sucessfully with upcoming challenges, because the production schedules and plans become ineffective after a short time on the shop floor. Established production planning and control systems are vulnerable to abrupt changes, unforeseen events and supply stock-outs in production processes and do not allow a real-time computation of sophisticated decision models [8], [16]. Furthermore, the increasing size and scope of central-planning-based manufacturing execution systems lead to rapidly growing structural complexity [6], [13]. Therefore, the 


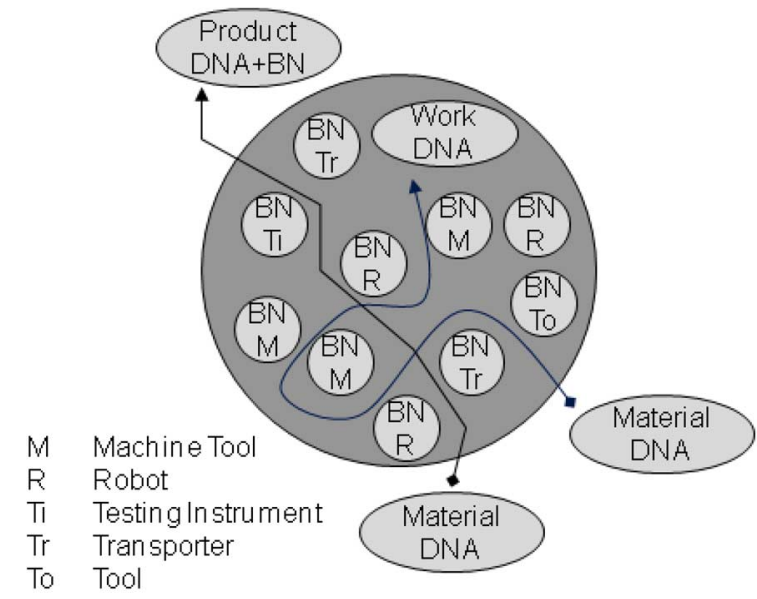

DNA genetic information evolving through generation BN in dividually achieved information

Fig. 3. Concept of BMSs [17].

shift to concepts such as mass customization and individualization encouraged the development of methods and concepts in agile manufacturing to cluster manufacturing systems into subsystems and modules [6]. Consequently, Reconfigurable Manufacturing Systems (RMSs) focused on rapid changes, modifications and disturbances in manufacturing systems [14]. The concept of RMS aims to provide scalable systems in response to market fluctuations as well as system and machine adaptability to new products. These capabilities are achieved by using modular software and hardware structures in the system (e.g., additional machines) and the machine level (e.g., machine hardware and control software) [15].

\section{B. Evolution of Planning Systems}

1) Biological Manufacturing Systems (BMSs): In the field of dynamic reconfiguration of manufacturing systems, the concept of BMSs was proposed in the second half of the 1990s [17]-[19]. BMS uses biologically inspired ideas such as self-growth, self-organization, adaptation and evolution. The single elements in a BMS, such as materials, machine tools, transporters, and robots are considered as autonomous organisms. The characteristics of each component within the BMS are represented by genetic information evolving through generational (called DNA type) and individually acquired experience during the lifetime of a system's element (called BN type) (see Fig. 3). The main focus of this concept is to deal autonomously with dynamic and unpredictable changes in internal and external production environments by changing a system's configuration. In order to implement the concept of a BMS, methods of evolutionary computation, self-organization, and reinforcement learning were developed [17], [18], [20].

2) Multi-Agent Systems (MASs): In MASs, centralized, hierarchical control architectures are replaced by a group of loosely connected agents. MAS mostly focus on the control level and on the concept of autonomous cooperating agents that operate on the shop floor. Thereby, each agent, which is a software entity,

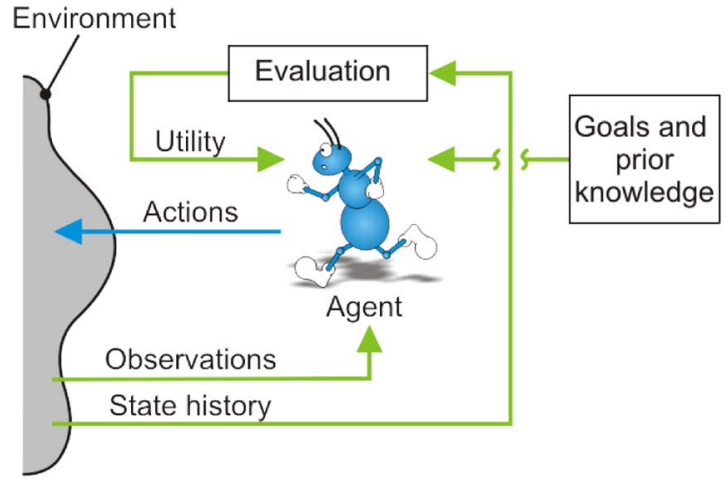

Fig. 4. The agent and its environment (according to [2] and [13]).

represents fundamental processing units (e.g., machines), orders and products [21]. Agent-based systems allow for a system structure with several distributed decision-making entities [22]. Monostori et al. [13] defined an agent as a computational system that operates in a dynamic environment from which it is clearly separated. An agent shows autonomous and intelligent behavior that comprehends, e.g., reasoning, planning and learning capabilities. According to Monostori [13] and Wooldridge and Jennings [23] an agent:

- is able to make observations about its environment (e.g., the physical world) and respond to changes that occur in it;

- acquires knowledge about its environment;

- chooses preferences with regard to the state of the environment;

- operates without direct intervention of humans or other external factors;

- initiates and executes actions in a goal-directed manner to change the environment.

The above-mentioned capabilities of agents and their integration in the environment are depicted in Fig. 4.

However, single agents have only a partial model of their environment. This can result from insufficient perception or a limited set of means for the acquisition and integration of new knowledge [13]. Hence, an individual agent does not have access to all the information that is required for an efficient decision-making process [24]. Therefore, a single agent has to coordinate its actions with those of other partners. When one agent cooperates and interacts with other agents in a network, a MAS is generated that achieves local and global objectives by dynamically communicating between autonomous modules [13]. Thus, production control strategies such as sequence planning, resource allocation and routing are negotiated between the software entities that act on behalf of their physical counterparts [24]. The negotiation processes for cooperation and conflict resolution are often solved with market-based approaches that are generally based on supply and demand as well as the respective priority of the order [8], [16].

The distributed responsibilities, tasks and resources entail a high robustness and adaptability to disruptions and reorganizations. In spite of changed boundary conditions (e.g., machine failure, reconfiguration of the production system), the principle 
interaction and cooperation between the different agents still takes place [24]. The efficiency of MAS is extensively dependent on exact and real-time information of their respective physical counterparts in the production environment [16]. On the one hand, this comprises resources as well as machines (i.e., subcategory of a resource) and, on the other hand, individual products [2], [11].

\section{Cognitive Factory Paradigm}

Recapitulating, fully automated production systems without human workers have been a convincingly viable vision for a long time, as pure automation was considered one of the key factors for implementing cost effective production. These systems can reach lead times and quality levels exceeding by far those of human workers. As this concept is quite feasible for reoccurring tasks, as found for instance in mass production, it disregards the immense cognitive capabilities humans possess and that enable them to react to unpredictable situations, to plan their further actions, to learn and to gain experience, and to communicate with others. While human capabilities turn mechanical workshops into the most flexible, adaptive and reliable form of production, they also cause very high production costs, especially in high wage countries, and thus are solely used for small series, prototypes or one-of production [4]. Hence, the realization of comparable cognitive capabilities in technical systems bears an immense potential for the creation of industrial automation systems that are able to overcome today's boundaries. A new paradigm in production engineering research pursues the objective of reaching the "Cognitive Factory," where machines and processes are equipped with cognitive capabilities that allow them to assess and increase their scope of operation autonomously [25]. In the following section, brief definitions of cognition and cognitive technical systems are given.

Cognition is the object of investigation of several scientific disciplines such as cognitive psychology, cognitive sciences, cognitive engineering, cognitive ergonomics and cognitive systems engineering [26], [27]. While robots have learned to walk, navigate, communicate, divide tasks, behave socially and play games [28], only a few examples for the application of artificial cognition in manufacturing exist. Therefore, several authors emphasize the need for cognitive systems to overcome deficiencies in automation [12], human-robot-interaction [29] and planning [30]. These Cognitive Technical Systems (CTSs) are equipped with artificial sensors and actuators, are integrated and embedded into physical systems and act in a physical world. They differ from other technical systems in that they perform cognitive control and have cognitive capabilities [25], [31].

Cognitive control comprises reflexive and habitual behavior in accordance with long-term intentions. Cognitive capabilities such as perception, reasoning, learning and planning (see Fig. 5) turn technical systems into ones that "know what they are doing." More specifically, a CTS is a technical system that can reason using substantial amounts of appropriately represented knowledge, learns from its experience so that it performs better tomorrow than it does today, explains itself and can be told what to do, is aware of its own capabilities, reflects on its own behavior, and responds robustly to surprise [32]. Technical systems that are cognitive in this sense will be much easier to

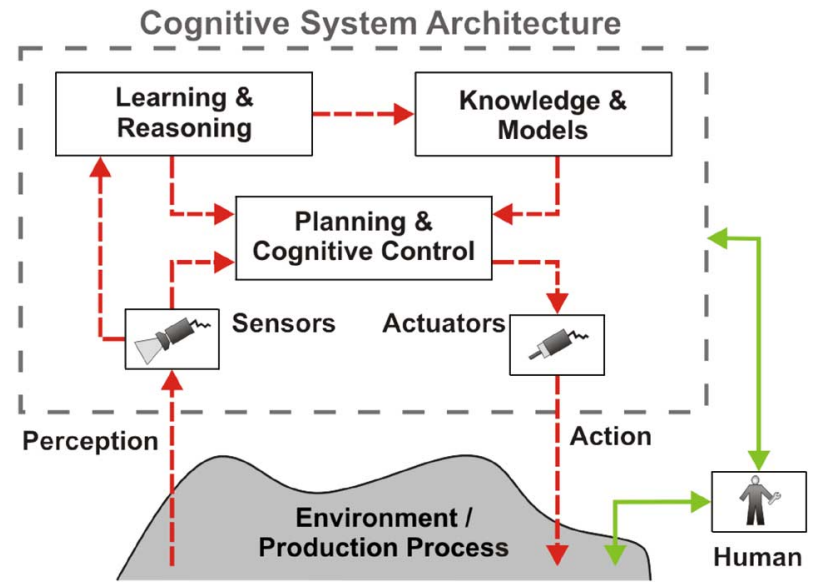

Fig. 5. Cognitive system architecture with closed perception-action loop [25].

interact and cooperate with and will be more robust, flexible and efficient. A factory environment, as a specific occurrence of a technical system, forms a superior field of application for artificial cognition, as it is full of concurrent, asynchronous and interactive control issues [10]. One of the key components of the Cognitive Factory is the cognitive perception-action loop. While performing their tasks, the Cognitive Factory acquires models of production processes, machine capabilities, workpieces and their properties as well as the relevant contexts of production processes. These models are continuously updated to adapt to changes in the environment and are then used to optimize action selection and parameterization [33]. Control systems that use predictive models of actions and capabilities achieve reliable operation and high performance quality [34]. To this end, the Cognitive Factory must be equipped with comprehensive perception, learning, reasoning, and plan management capabilities [25].

The paradigm "cognition" in terms of the factory denotes that machines and processes are equipped with cognitive capabilities. In technical terms, this comprises sensors and actuators that enable machines and processes to assess and increase their scope of operation autonomously [25]. So-called "cognitive sensor networks" allow for real-time acquisition of production and process data and a suitable feedback to the process control. Models for knowledge and learning equip the factory with information about its capabilities and help to expand the abilities of the machines and processes. Continuous information about the status of certain actions and production steps allow machine parameters to be improved without human intervention and enable an autonomous maintenance based on prediction. Confronting the Cognitive Factory with the task of producing a new product, its elements "discuss" this task and decide whether or not the product can be produced with the available production resources and abilities, thus recognizing its own limitations. By storing previously defined strategies within a database along with mechanisms of information processing and retrieval, the system is able to derive further decisions [4]. The Cognitive Factory is able to learn from previously performed tasks and is, therefore, easily adaptable to new products, thus enabling small lot size and individualized production at a competitive 


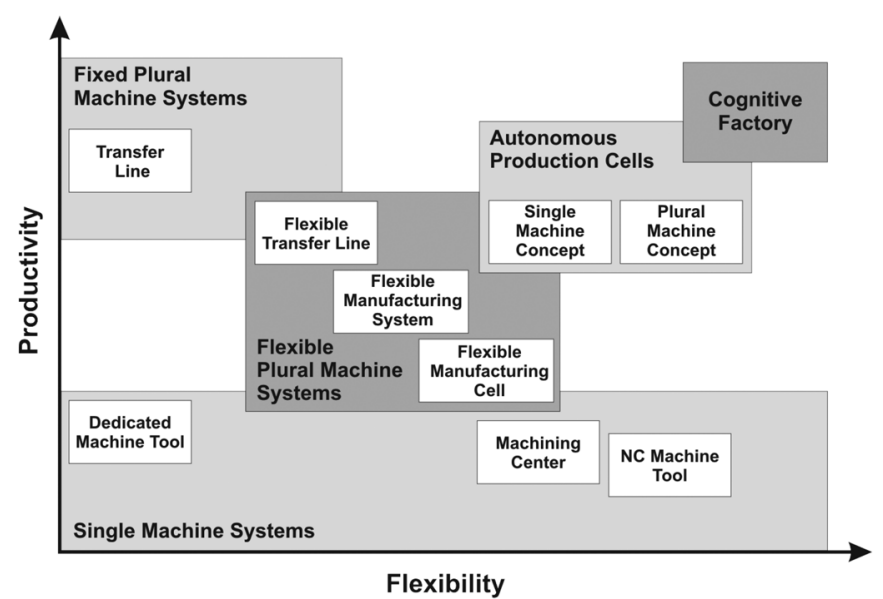

Fig. 6. Classification of manufacturing systems and the incorporation of the cognitive factory [25].

cost level. Because the Cognitive Factory knows its own capabilities, process planning, which today is performed primarily by humans, will to some extent be carried out directly on the shop-floor level and will allow to begin production with rather raw information and without detailed advance planning [4].

Using self-maintaining systems, the Cognitive Factory will minimize the efforts and the time needed for reconfiguration and setup and reduce the down time of factory environments. The overall goals of the Cognitive Factory can be summarized as follows:

- making the knowledge available in factory systems more transparent;

- enforcing the interconnection between men and machines;

- creating manufacturing systems that are more adaptive than existing ones;

- and enabling autonomous planning processes.

The Cognitive Factory combines the advantages of automated systems (e.g., low costs, high quality, high efficiency, and low manufacturing times) with the flexibility, adaptability and reactivity of common human workshops (see Fig. 6).

\section{Production PlanNing AND CONTROL}

\section{A. Product-Based Production Planning and Control}

1) State-of-the-Art and Research Gaps: In recent years, research work was focused especially on the increase of flexibility and adaptability in production environments by acquiring and using information from resources and machines. Essential information of the individual product (e.g., quality) was barely utilized [2], [11]. In most production environments, the workpiece flow is passive and is handled or transformed only by active resources [35]. However, it is inevitable that the individual project is considered in order to improve flexibility in production management [36]. A further step to increase the autonomy of production systems and to realize autonomous products is the active consideration of the product in the production control and the storage of current product-specific information directly on the product. This includes the availability of product state, product location and especially the consideration of quality information in the production process. Thus, the effectiveness of
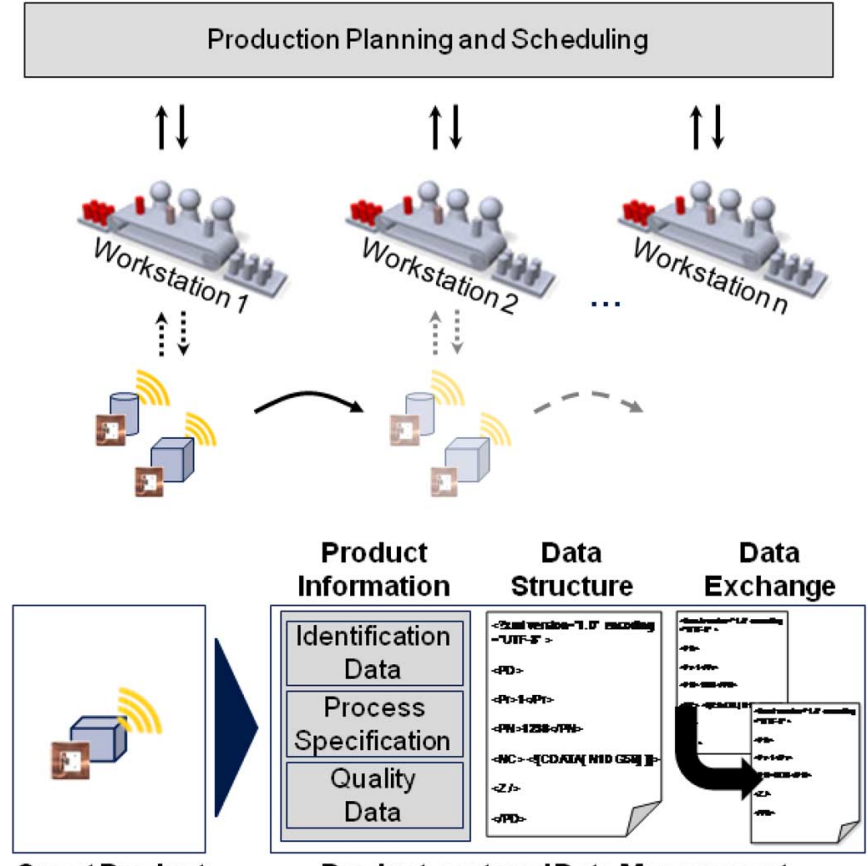

Smart Product

Product-centeredData Management

Fig. 7. Schematic illustration of the product-based control of production processes [38].

production planning and control systems can be improved unambiguously. The availability of current product information is therefore a supplement to existing production information. The individual product can therefore be introduced as an additional element of control in the overall control structure of a production system. Hence, instant changes both in product states and in manufacturing systems can be considered for an adaptive production control [2].

2) Approach: As a prerequisite for introducing the product as an additional element of production control, reliable access to relevant product-specific information, consistent assignment and the regular update of the product's state must be ensured. The utilization of RFID technology provides the basis for a precise image of the real world and supports autonomous decision-making in distributed and automated production control [24]. RFID shows several advantages compared to other Auto-ID (automated identification) technologies (e.g., barcode) such as automatic and wireless machine-to-machine communication, or the dynamic and automatic storing and retrieving of information. Thus, items can be tracked automatically and critical product-specific information can be stored directly on the product [10], [16]. By combining workpieces or products with RFID transponders, they become "smart products." They provide the means to share their information with the planning level and with other resources through a respective communication infrastructure. Hence, smart products can automatically process received requests and respond to the available RFID readers [37]. Smart products are therefore able to actively influence their own production, distribution and storage [24]. The communication between the smart product and resources in the course of the production process as well as the product's integration in the overall process is depicted in Fig. 7. It 

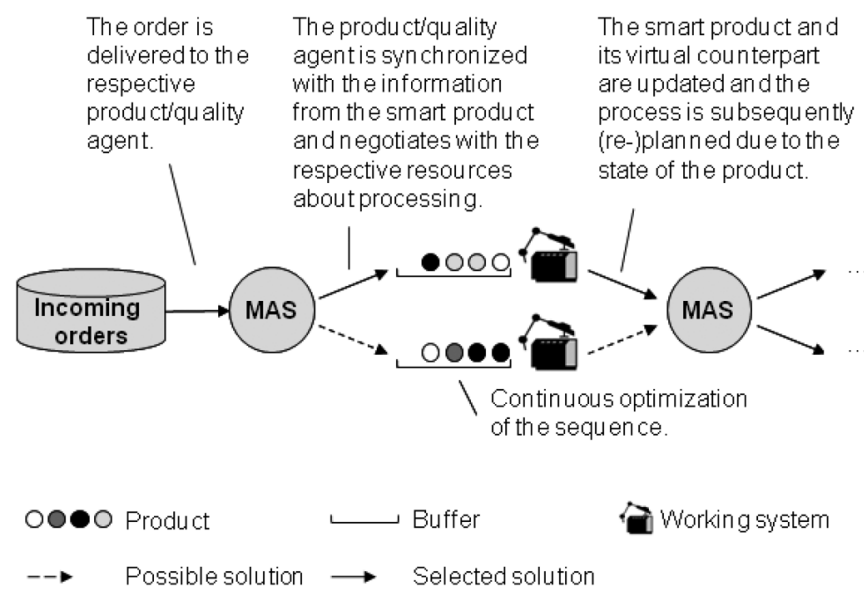

Fig. 8. Exemplary multi-agent supported production process [2].

includes the continuous monitoring of smart products and resources (e.g., state information, operational availability) and the respective transfer of the updated information from the shop floor to the planning level. Product-centered data management is a necessary prerequisite for a decentralized product-based control of production processes. It consists of the respective definition of crucial product-specific information that needs to be stored on the smart product. This information needs to be logically structured on the RFID transponder, in order to ensure reliable and standardized processes as well as an easy exchange and modification of product-specific data. A standardized XML schema was developed for the representation of product-specific data, including identification data, processing data and quality data. The detailed approach is extensively explained in [2], [11], and [38].

As stated before, the single agents in a MAS are excessively dependent on updated real-time information about resources and products from the shop-floor, thus providing an optimal performance of production planning and control. Specifically, the requirements that emerge are associated with product customization, traceability and information management. Hence, the observability, quality, availability and productivity of the production system can be enhanced with improved efficiency of response to disturbances in process control. By using the above-mentioned smart product, an applicable real-time information exchange between a product in the production environment and its virtual counterpart in a MAS can be realized [2].

Each master workpiece is equipped with an RFID transponder, on which critical product-specific manufacturing information such as the job number, the initial work plan, quality data and the production history is stored. Fig. 8 shows an exemplary multi-agent supported, product-based production process. The proposed MAS consists of planning agents, product agents and machine agents for the planning and control of production processes [2].

Based on incoming production orders, the planning agent releases every order for the production process and transfers the individual order to the product agent. Beyond the job release, the planning agent must further consider potentially altered boundary conditions (e.g., machine breakdowns). The product agent is in charge of the subsequent production of the workpiece/product and the respective throughput in the production environment. It must consider the specified work plan, verify machine capability and negotiate the processing with the machine agents. Furthermore, the product agent that is concurrently a quality agent has to evaluate feasible deviations between planned and executed processes and the respective workpiece quality [2]. The machine agent negotiates with the product/quality agent and balances the request for processing with the available resource capacities. Furthermore, the machining sequence is optimized with the new addition of a production order [2].

Decentralized product-based data management allows for a direct and error-resistant interaction between automated production processes and feasible manual interventions of human workers such as rework of workpieces. The complete documentation of executed process steps and quality information with the continuous update allows for the direct consideration in subsequent production processes and feasible modifications (e.g., transposition of products, rework). Thus, necessary production processes can be determined automatically based on the designated workflow and its current state in the manufacturing environment. The individual agents are therefore able to access, manage and utilize the information carried on the RFID transponders and apply it to production planning and control despite the feasible manual interventions of human workers [2].

In the case of manual tasks, such as assembly processes or rework, the workpiece can be removed from the automated production process. The human worker can use product-specific information as work instructions. After finishing the manual work steps, the operator can document his work on the smart product and return it to the automated production cycle. With standardized, product-centered data management, the smart product can be automatically reintegrated into the control of the MAS. In the same modality, it is possible to manually dispatch a production order (push order) to the system. Thus, unprecedented levels of flexibility can be accomplished in production environments with a combination of automated and manual processes [2].

3) Results: Parts of the approach explained above were prototypically implemented in a Flexible Manufacturing System (FMS) that serves as a demonstration platform, which includes substantial elements and capabilities of an industrial production system.

The original control platform of the FMS is characterized by a hierarchical architecture. The production processes for the fabrication of products are completely predefined and controlled by a central computer. This includes the explicit presetting of manufacturing parameters and robot or CNC programs. In case of an unforeseen event, the process stops immediately and the system displays an error that has to be solved by human intervention.

The FMS is used as a demonstration platform to replace conventional centralized decision-making systems with distributed decision-making entities. In a first stage, the approach described above was prototypically established for the product-based control of production processes. This includes the RFID-based transfer of manufacturing information between the smart product and the CNC-based machine tools, as well as the exchange of routing instructions with handling robots, storage and 
the pallet transport system. For the experimental setup RFID systems with $13.56 \mathrm{MHz}$ (High Frequency, HF) and passive RFID transponders were chosen. HF transponders usually have more memory than Ultra High Frequency (UHF) transponders. Furthermore, it is easier to have a limited and distinct communication area with high frequency RFID systems [39].

As a precondition for the product-based production control, the centralized control structure of the original system was replaced by developed heterarchical control entities. The appropriate control software for the local cells establishes a communication between the particular RFID system and the respective workstation, robot and local computer.

The process sequence is exemplarily explained with the handling and the subsequent machining of a workpiece. When a workpiece arrives from the pallet transport system, the loading robot transports the pallet with the respective workpiece to an intermediate storage facility. At this station, the XML file on the RFID transponder, which is attached to the workpiece, is read and the data is assessed by the corresponding subsystem. Throughout this operation the subprocess, the prerequisites for the execution and the verification of the respective machine are evaluated using the above described methodology. In case of the acceptance for the subprocess, the respective product-specific data for processing (e.g., CNC code) is transferred from the transponder to the specified CNC machine tool of the corresponding work cell. At the same time, a direct communication between the smart product and the robot control is established, to initiate the transportation and the required handling operations for the transfer of the workpiece to the respective $\mathrm{CNC}$ machine. During processing, the control software monitors the CNC machine for potential errors as well as for process documentation purposes (e.g., duration of machining). The collected data is used to update the smart product after processing and to delete nonrequired data in order to improve resource efficiency. After completing the subprocess, the part is unloaded from the machine and the data on the RFID-tagged object is subsequently modified to the new state. The information is evaluated with respect to whether the product has to be processed again on the other CNC machine or whether it can be transferred to the next station (e.g., assembly) [2].

\section{B. Simulation-Assisted Production Planning and Controll}

1) Motivation: Discrete-event simulation has proven to be an effective and efficient software tool to assess future strategies in production planning and control with regard to their effectiveness and efficiency [40]. Traditionally, the simulation of manufacturing systems was used to support the decision making in strategic problems, e.g., as a tool for testing a newly designed factory layout before building it [41]. But it is becoming more and more common to use simulation in the operating phase of a factory as well. For the operational simulation an existing production system is modelled with its dynamic workflows within a software tool in order to consider stochastic effects like breakdowns and interrelations between different workflows for making prognoses regarding the future system behavior. The models are then used to support production planning and control (PPC). The real-time control via online simulation allows for a visualization and identification of problems in shop-floor control in real time [42]. This type of simulation presumes that simulation models are highly detailed they have a longer lifetime than models from traditional simulation studies [43]. Furthermore, they demand that the effort to update the model in accordance with the real system can be done in a time and cost effective way [43].

2) State-of-the-Art and Research Gaps: In simulation experiments, data acquisition and simulation modelling belong to the most time consuming tasks. For this reason, many authors mention the necessity to improve the efficiency in these areas (e.g., [40] and [43]-[45]). In literature especially two approaches are being discussed:

1) reducing the complexity of simulation models,

2) generating simulation models to a large extent automatically.

The first aspect is discussed for example in [46]. Aderson and Olsson [43] use a standard discrete event simulation package for supporting operational decisions regarding daily labor assignment in a customer-driven assembly line. The information for building the simulation model is gathered by interviews and time data collections. They propose an automated daily update of the simulation model with the real status of the assembly line every morning, but due to a missing data system this was not implemented. Lulay [40] uses the operational simulation for the coordination of partly autonomous production structures towards a common objective with a hierarchical model. Due to the complex model structure and the long response times a general usage is not suggested by the author. Lulay implemented interfaces to production planning and control system for updating the actual workload. The structure and organizational data, however, is not updated automatically.

In order to compare different strategies in production planning and control via operational simulation, the simulation models have to be easily adaptable to changes in the production environment [40]. In this context, Weigert et al. [47] develops simulation models that are able to adjust to the real production system. He regards models for operational simulation no longer as static objects, but allows for change in status, parameters and structure at any time. He divides the adjustment into the synchronization with the system status (e.g., workload) and adaption to changes in the model parameters. For this automatic and continuous adjustment, the data provided of the production data acquisition is applied. Later, Weigert et al. [45] develop a concept for auto-generating simulation models in the semiconductor industry from databases in enterprise resource planning (ERP) and manufacturing execution systems (MESs) based upon so called mini-models. It includes the process flows and structure as well as the parameterization of the model. Afterwards this model is used for finding an optimal schedule [48]. The applicability of the simulation system to support production planning and control depends among others on the conformance of real-world decision strategies to the ones simulated. These strategies often depend on the decisions of human operators [49] and thus are not explicitly known. Milberg and Selke [44] developed a concept to integrate the identification of human strategies in production control into the automatic generation of simulation models using a statistical comparison between the theoretical patterns of different strategies with the actual production data. This allows for an improvement 
in the correctness and reliability of the generated simulation models. An important aspect in this context is the identification of the implicit strategies in production planning and control. $\mathrm{Li}$ and Olafsson [50] present a methodology for the derivation of scheduling rules using data mining directly to production data. They focus on a single machine problem with a jobwise comparison of jobs. The approach can be extended easily to large and complex production environments, but there is still research work to be done in order to consider comparisons between multiple jobs.

In conclusion, it can be stated that although important progress has been made in the generation of models for operational simulations, there are still plenty of problems to be solved. One aspect is the graphical representation. It is an important aspect to achieve a better acceptance, but it is mostly not considered in the automatic generation of simulation models. The second aspect is the data needed for the model generation. Due to reducing costs of data aggregation and storage, the availability of data is increasing. This is the same case in manufacturing, where more and more MESs are installed and hence data regarding product quality, equipment efficiency and material flow are recorded. Shop-floor control, however, has received little attention from the data mining community [51]. The analysis of the production data still offers a large potential for improving the automatic generation of simulation models, especially regarding the identification of implicit human strategies in production planning and control. Another problem is the quality of the available data. This often contains lots of missing or incorrect values.

3) Approach: In order to overcome the deficiency in the interpretation of the available production data for the identification of implicit strategies, a method for recognizing strategy related patterns has been developed. For this purpose, underlying data from Production Data Acquisition (PDA) is analyzed to identify patterns and to recognize human strategies and provide them for an automated generation of simulation models. In a first step, the data logged by the PDA is preprocessed in order to modify the available data and to extract the parameters of the different jobs needed for pattern recognition. Subsequently, a ranking describing the parameter values in comparison to the other jobs available in the same decision is introduced. This ranking is then used for the identification of the most commonly used strategies. Based upon this information, a regression analysis is used for recognizing the order of their application. Finally, the degree of fuzziness in the application of the identified strategies is analyzed. The usage of the proposed methodology will improve the ability of companies to predict the behavior of their production upon a changing and uncertain environment and hence can be a valuable tool to encounter today's challenges of producing companies.

4) Results: The first task is the extraction of appropriate features for the identification of strategies in PPC. The presented approach assumes, that human strategies can be described by combining different priority rules like earliest due date, customer priority or minimal slack time. Given this assumption, for each strategy a directly related feature can be found. Sometimes it is already part of the data stored in the SQL-database (e.g., due date) or it has to be calculated from existing data like for instance the slack time as the difference between remaining
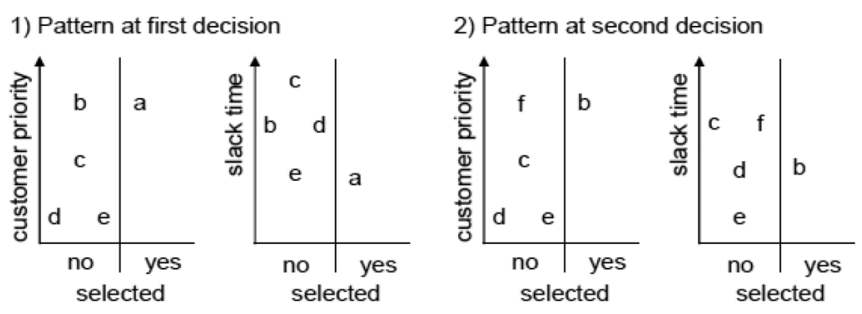

Fig. 9. Patterns in time discrete depiction.

time to the due date and remaining processing time. Nevertheless, the identification of patterns in production control has to deal with two major problems:

1) A human scheduler does not necessarily apply only one priority rule, but may use a combination of different ones.

2) A production is a dynamic environment and thus the sample of lots is changing from one decision to the next, since new lots may enter a buffer and others may already be processed.

This makes it necessary to look at discrete points of time, where the decisions are made. To obtain analyzable patterns, the selected lot is compared to the remaining ones. If different sequencing strategies are applied simultaneously, the assignment between pattern and strategy is complicated and has to be derived regarding a large sample size. Fig. 9 shows an example for patterns occurring when different strategies are applied and additional lots enter the buffer in between the first and the second decision. A more detailed description of the feature extraction can be found in [49]. These patterns have to be found in the preprocessed data. In order to achieve this, a ranking has been introduced, describing the priority of a job regarding the related parameter [49]. Regarding solely the jobs selected for processing in the next step, it is assumed, that the rankings of parameters related to frequently applied strategies in average are closer to one than the ones used less often. This implies, that the lowest sum signals the mostly employed strategy. Subsequently, limits for the application of the identified strategies are derived via a regression analysis. Finally, the order of these strategies is retrieved by minimizing the difference between applicability and application of the identified strategies. In the last step the applicable tolerances have to be identified. This means, that for a scheduler it is important for example whether the slack time is three or four days. But it is not important, whether it is three days and four hours or three days and five hours. The tolerance range is detected by comparing the absolute parameter value of the selected lot and the one with the ranking 1 . The tolerance is estimated by the quantile of the differences, in order to exclude extreme variations.

\section{Self-Optimizing Cell-Control}

1) Motivation: The cell control comprises the levels 2 and 3 of enterprise control systems according to [52] and deals with the dispatching of the production, the detailed production scheduling, and the control of production processes. A successful detailed production planning on the shop floor is one core factor to reach a high supplier's reliability, thus leading to satisfied customers, and is therefore the basis for a long-term economic success. 

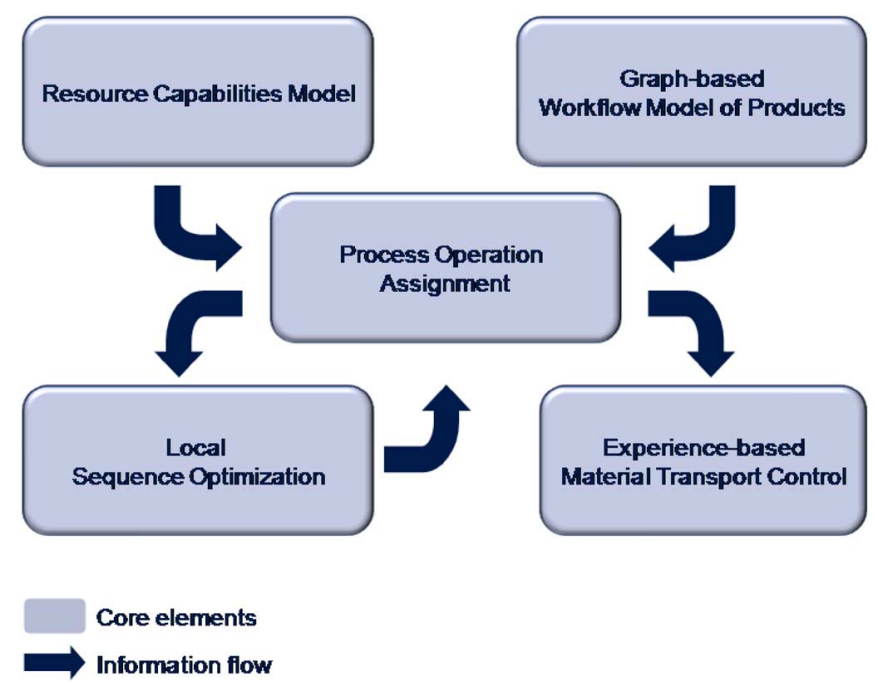

Fig. 10. Core elements of the self-optimizing cell-control.

2) State-of-the-Art and Research Gaps: Within the past years, several approaches for an efficient cell control were developed, which may be divided into two basic principles: On the one hand, methods for an efficient job scheduling in production cells, using given production workflows as an input have been developed [6], [53]. On the other hand, research work has been accomplished, in generating workflows from a set of small tasks, so called action primitives [54], [55]. One of the concepts of the first type are the agent-based approaches with a strong focus on job scheduling like Holonic Manufacturing Systems [13], [21], [56]. These agent-based control systems have reached a high degree of maturity and are already applied in industry [57]. Although these concepts have proven to react highly efficient to machine disturbances and rush orders, they are not able to consider past experiences and improve workflows during runtime. A different approach is used in the concept of Autonomous Production Cells, where single fractions of a factory are organized as a single company within the company. However this concept is restricted to methods of enterprise organization and does not focus on control systems [58], [59]. The second group of approaches address the control of single robot cells by the use of action primitives (e.g., [54], [55], and [60]). An early concept for control systems that regards machine capabilities has been introduced in the Intelligent Manufacturing Systems Program (IMS) [61]. However, existing projects focus on special problems in the production control of factory cells. A concept to integrate the planning and control of workflows and transport routes, which regards machine capabilities and improves itself during runtime is not known.

3) Approach: A concept developed by authors of this paper consists mainly of five core elements: 1) a graph-based model of production processes; 2) a description model of resource capabilities; 3) a control module for the allocation of process operations to resources; 4) a learning sequence optimizer on a local (i.e., resource) level; and 5) an experience-based material transport planning algorithm (see Fig. 10).
The interplay of these five core elements and the corresponding information flows can be outlined as follows: A prerequisite to implement planning and learning algorithms is a sound data model of process operations of the products to be manufactured on the underlying production system. This graph-based model contains the required process operations of a product during its production process. A data model for the adaptive control of production operations has been developed and tested on a factory cell. The graph-based representation of the approach distinguishes four main elements: 1) product; 2) process operation; 3 ) process operation time; and 4) process plan. A product is defined as one unit of a good that could be produced on the underlying production system [62]. Therefore, this term is used for end products as well as for assemblies, subassemblies, and components. An operation is, in accordance to [62], an activity that could be included into a process plan. The time required to complete a process operation is called process operation time. It often depends on its upstream process operations [63]. For every product, a process plan is required for its production. The process plan represents a precedence graph of the process operations, including sequence constraints and is therefore equal to a cycle-free digraph. Thereby, the set of vertices $V$ represents all process operations required to produce the respective product $p_{i}$. The set of edges $E$ are the executable changeovers from one process operation to another, which represent the process operation times required for the execution of the process operation. An additional requirement for an autonomous allocation of jobs to resources is to equip the planning algorithm with knowledge on the capabilities of the resources of the production system. The resource capabilities are hereby described as a set of process operations according to [64]. The full set of operations that can be performed by a resource is called a profile. This profile is published to the assignment module, and serves as input for the job allocation algorithms, which enable the control system to share jobs among its resources and to distribute tasks between them. Following the assignment of jobs to resources, the sequences of the devolved process operations have to be optimized. The optimization on a local level is hereby conducted by graph-search algorithms. During runtime of the production system, the realized process operation times and their quality results are transferred back, to update the process model and to support midterm adaptation of production workflows. Finally, the material needs to be transported to the resources in order to accomplish fabrication and assembly tasks. Despite existing approaches, this task is not merely performed purely deterministic on the basis of known and started jobs, but transports are started anticipatorily based on previous material calls. This predictive transport control comprises four steps:

1) Estimation of time for material calls: For every resource of an underlying production system, the corresponding time of material call for every product are estimated according to the past material calls. This is denoted in the following equation:

$$
\tau_{p_{i}, r_{l}}^{t+1}=\frac{1}{n} \sum_{n=1}^{n} \tau_{p_{i}, r l}^{t}
$$


where

$$
\begin{array}{ll}
\tau_{p_{i}, r_{l}}^{t+1} & \begin{array}{l}
\text { time of material call for product } p_{i} \text { at resource } \\
\\
r_{l} \text { in period } t+1 ;
\end{array} \\
n & \text { number of past material calls. }
\end{array}
$$

2) Decision making: The estimated time of material call is used in the second step, to decide, whether a material transport is started or not. The according decision function is denoted below

$$
V\left(p_{q}\right)=\pi c_{1} t_{\text {prod }}\left(p_{q}\right)-(1-\pi) c_{2} t_{\text {trans }+}\left(p_{q}\right)
$$

where

$\begin{array}{ll}V\left(p_{q}\right) & \text { value of a possible transport; } \\ c_{1} & \begin{array}{l}\text { cost factor for gained production time of } \\ \text { accepted transports; }\end{array} \\ c_{2} & \begin{array}{l}\text { cost factor for transport times (including } \\ \text { transport back) of not accepted }\end{array} \\ & \text { transports; } \\ \pi & \begin{array}{l}\text { probability for the acceptance of a } \\ \text { transport; }\end{array} \\ t_{\text {prod }}\left(p_{q}\right) & \begin{array}{l}\text { production time gained by an accepted } \\ \text { transport } p_{q} ;\end{array} \\ t_{\text {trans+ }}\left(p_{q}\right) & \begin{array}{l}\text { transport time (including transport back) } \\ \text { of not accepted transport } p_{q} .\end{array}\end{array}$

3) Transport: A transport is started, if the value of the above equation is positive.

4) Transport approval: Once a transport arrives at its destination, (i.e., a resource), the control system checks if the material of the transport is required at its destination. If yes, it is used for production, if not, it is transported and stored again. The result of the check is transferred to the decision module and updates $p_{i}$ in the above equation.

4) Results: A full concept for a self-optimizing cell control has been developed. This comprises the definition of a data model that includes product requirements as well as resource capabilities. In order to provide high applicability, the data model is strictly in line with existing guidelines. In addition, a full model for a predictive transport control has been developed and introduced.

\section{Automated Processes AND Operations}

\section{A. Machining Planning}

1) Motivation: In order for companies to remain competitive in a global market, design and manufacturing must be better integrated [65] and increased flexibility and dynamic adaptation in manufacturing environments must be provided [66]. A strong market driver is the need for the fabrication of customized, individual and even engineered-to-order parts that require methods and tools for flexible and autonomous fabrication planning, without the need for time-consuming, manual replanning, and reprogramming. Planning the fabrication process for new designs autonomously requires using a generative approach, generating the process plan from scratch and avoiding human interaction in the planning process. This results in a highly complex task to analyze the design and derive the required fabrication information [67]. Current process planning for machining is carried out offline and machines only execute the generated plan. Enabling machines to know their capabilities as well as plan and execute their own fabrication processes will allow for wider part flexibility and reacting to unforeseen events on the shop floor. To enable the execution of manufacturing operations in a real manufacturing system, multiple layers of knowledge must be integrated [68] since planning for the manufacture of parts involves symbolic computation and extensive geometric reasoning [69]. To enable online feedback from the shop floor to the planning system, a perception system must be integrated. The approach, that is presented, addresses the rapid fabrication of customized or individual parts using multiple CNC machine tools and handling devices within a FMS.

2) State-of-the-Art and Research Gaps: The state-of-the-art in Computer-Aided Process Planning (CAPP) are feature-based approaches that encapsulate the semantic knowledge of manufacturable shapes into smaller units within parts, thus making them accessible to a computer-based method. An example of a system able to autonomously derive all manufacturing information from a design and to fabricate the part is the internet-based CAD/CAM-system CyberCut [70]. The system allows the design of parts by means of manufacturing features in a $2.5 \mathrm{D}$ environment. From this design information, the machining instructions are derived in a three step process from macroplanning to microplanning to toolpath planning. The part is then directly machined on a three-axis milling machine. This design-by-manufacturing-features approach reduces the necessary transition between the domains to a fixed mapping able only to statically represent machining capabilities and tools and their relation to predefined features. However, the mapping from one domain to the other by feature recognition (i.e., recognizing domain specific features only from geometric information) or by feature mapping from a feature model still remains a difficult problem [71]. Feature recognition approaches are still bound to specialized application areas or specific part families [65]. Since predefined transformation rules that describe the decomposition of design features into machining processes are difficult to handle and to maintain [71], the mapping must not prescribe the decomposition but the underlying principle of how to decompose design features into viable manufacturing processes. To enable a design-to-fabrication system to reason about its task and to create a process plan autonomously, it must be equipped with knowledge models. Shah and Mäntylä [72] classify the necessary knowledge models into the hierarchy of universal, shop-level, and machine-level knowledge. One approach to embedding knowledge in the system is the use of an ontology [73], [74]. If the ontology is logic-based, it is possible to reason directly about alternative production plans and capabilities using the knowledge base. This is appropriate for the universal knowledge level, i.e., knowledge common to any manufacturing system, and shop-floor level knowledge, i.e., knowledge for a particular machine shop, due to its symbolic nature. However, machine level knowledge for fabrication machines, and in particular CNC machines, is highly coupled with geometric models, constraints and transformations, and thus is not 
well suited for description through an ontology. For example, a specific difficulty is the mapping from the model level description of a part down to the application level, thus transforming a part description into a process plan that can be executed on the machine level. Promising approaches such as STEP-NC [75], [76] for micro- and toolpath planning have only reached limited industrial uptake and still have issues in implementation [77].

Therefore, the authors propose that another kind of knowledge representation is required to satisfactorily encode machine level knowledge for automated generation of machining plans. The goal is to do this in a general and flexible way for macroplanning right down to toolpath planning [78].

While formal grammars primarily work symbolically, shape grammars work in the domain of geometry [79]. With rules and basic elements from points to solids [79], [80], general geometries can be represented and generated. The advantages of shape grammars are that they can represent knowledge about both form and function, since they represent geometric shapes in addition to symbols [81].

Engineering grammars have been proven able to represent and generate designs for Microelectromechanical Systems (MEMS) resonators [82], motorcycles [83], gear systems [84] and also to integrate knowledge on fabrication [85], [86], to translate designs into fabrication ready models. Furthermore, approaches generally based on grammars and algebras for translating designs from CAD models to manufacturing information and process planning have been proposed in the areas of CAD modeling [80], process planning for turning [86], and generative CAPP [87].

3) Approach: The process, from design to fabrication, is shown in Fig. 11. It begins with the geometric models of the design and the raw material. With a Boolean subtraction operation, the Total Removal Volume (TRV) (i.e., the volume, which needs to be removed from the raw material to yield the finished part) is generated. Using fundamental knowledge of the machining process, material (i.e., volume) is removed from the TRV by applying rules of the shape grammar using the knowledge encoded in the rules and the vocabulary, simultaneously instantiating the toolpath and the necessary CNC instructions to carry out the operation on the machine tool. After the rule application, the TRV geometry is updated and further rules are applied until the volume of the TRV is minimized. Once the volume of the TRV has been minimized, the CNC instructions can be collected from the created toolpaths in the sequence of the rules applied. The resulting machining plan can be executed on the machine tool. The Removal Volumes (RV) contain also as labels the parameters of the RV that are instantiated during rule application and the definition of the used machine tool, selected tool, toolpath, and machining instructions. The complete machining instructions can then be collected from a sequence of RVs. The machining process knowledge is encoded in the rule set and in the vocabulary. In the vocabulary, the elemental shapes that can be removed by a machining operation are generated from the combination of the tool shape and the kinematic capabilities of the machine tool from the machine tool library. The vocabulary consists, in general, of the Removal Volume shapes, toolpath-curves and endpoints of the toolpath, the shapes of the part to be fabricated, the raw material and the TRV. The labels allow

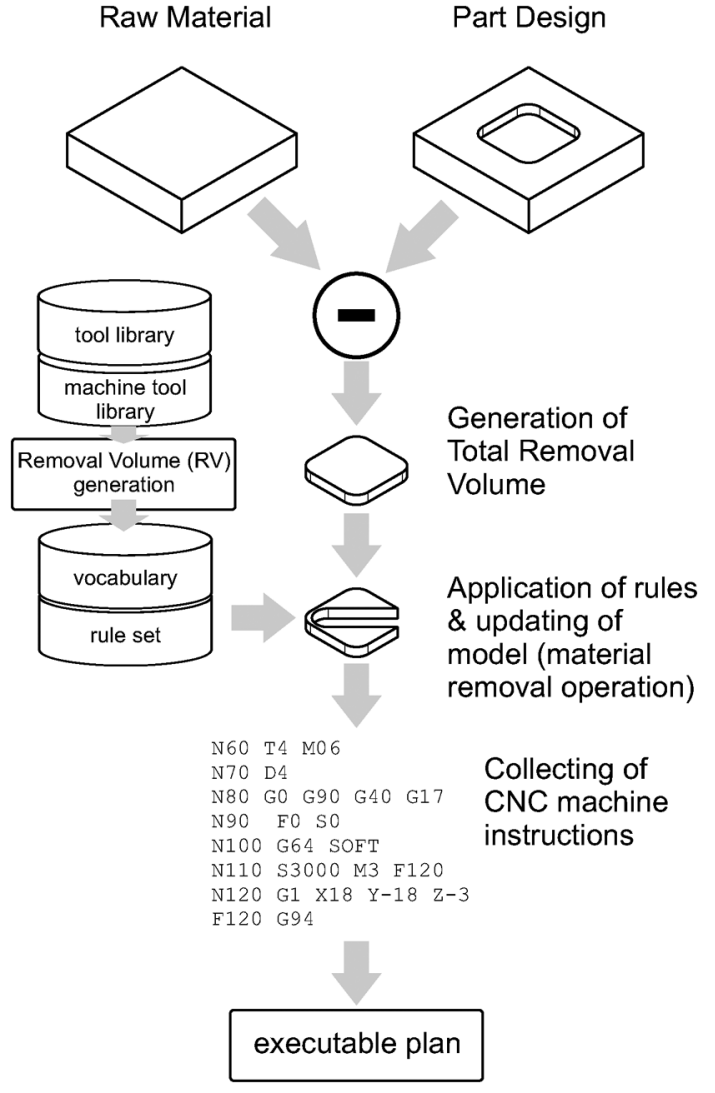

Fig. 11. Design-to-fabrication process.

further control over the grammar. The set of rules consists of the fundamental rule to apply an RV to the TRV and of auxiliary rules for setting the initial tool-path origin, repositioning of the tool and approaching the raw material.

The TRV represents the Initial Shape of the grammar. During the application of RVs to the TRV, the TRV and the sequence of $\mathrm{RV}$ applications represent the working shape. The details of the specific shape grammar for machining planning can be found in [78] and [88].

When the machining planner has decided which operations need to be carried out and identified a selection of adequate machines, classical planning is used to create a sequence including transportation and handling operations. This sequence is called the Executable Plan (EP), which can be fed into low-level machine controls.

Classical planning finds an action sequence that, starting from a defined initial state, achieves a given goal state. It employs a logical model consisting of predicates and entities. The Planning Domain Description Language (PDDL) [89] is used in the approach presented. A multitude of open-source planners are available that support the planning based on a domain description and an accompanying problem description. The domain describes the predicates and actions, while the problem describes the specific setup and entities as well as the goal of the single planning task. A concise formal definition of planning can be found in [90]. The machining operations are modeled in PDDL in analogy to assembly operations. The execution of a machining operation corresponds to adding a virtual part to the 
product in the planning model. The added part represents the CNC instructions to run on the machine tools.

Inclusion of an adequate perception mechanism into the cognitive system provides visual feedback, allowing for monitoring of the system's state, indirectly through the product state, possible failure diagnosis and consequent recovery. To build an integrated cognitive manufacturing system, a variety of ad-hoc perception mechanisms is used, for example:

- a machine vision-based approach using a Dark Field Light and edge detection algorithm;

- 3D laser scanning based on point-to-surface distance measures for deviation estimation.

The selection of an appropriate mechanism depends on the type of the examined part and the features to be inspected.

Since the machining planning is a deep knowledge, bottom-up approach, only machining specific information is generated. Such a plan, however, is not directly executable on the complete manufacturing system because it lacks information on logistics and transportation of the parts, which are outside of the machining planning scope. To enable the execution, the plan has to be completed with this information. To do so, the machining planning is interfaced with a global, abstract planning system. Fig. 12 shows the three areas of the global and local planning: the global input and output, the global planning and the local planning. The overall planning process is initiated by defining the global goal to produce a part $\mathrm{X}$ and passing the part designator to the global planning system. Designators are partial part descriptions (e.g., material, dimensions or CAD model) that are updated in the execution loop used to parameterize the system's actions.

In the global planning system, a reference model and raw material are found. Furthermore, the availability of capable machine tools and their tools is estimated. Afterwards, a raw material designator along with the original part designator is passed to the local planning system. There, the TRV is calculated and the Initial Plan (IP) for machining is generated. To complete the IP, and thus create a feasible and executable plan, the IP must be manipulated by the global planning system. To enable this manipulation, the domain specific IP must be generalized (i.e., abstracted by segmenting it into single operations).

The machining plan is segmented into operations, each separated by machine tool changes, tool changes and repositioning movements at rapid traverse. After the abstraction, the Abstracted Plan (AP) can be accessed by the global planning system. Each operation in the AP is fully defined by the tool, machine tool, CNC instructions, cost/time information and the expected resulting shape of the TRV and the part. Providing geometric models of the part shape is necessary to infer the interrelations of the different operations and to enable feedback from perception. The AP is passed to the global planning system, where it is analyzed. The global planning system adds missing process steps such as transportation operations. Furthermore, the sequence of execution can be altered, depending on the interrelations of operations and online feedback from the system. The final result of the planning system is the Executable Plan (EP). This plan can be executed in the manufacturing system, depending on the current state and system feedback obtained from the perception component, involving

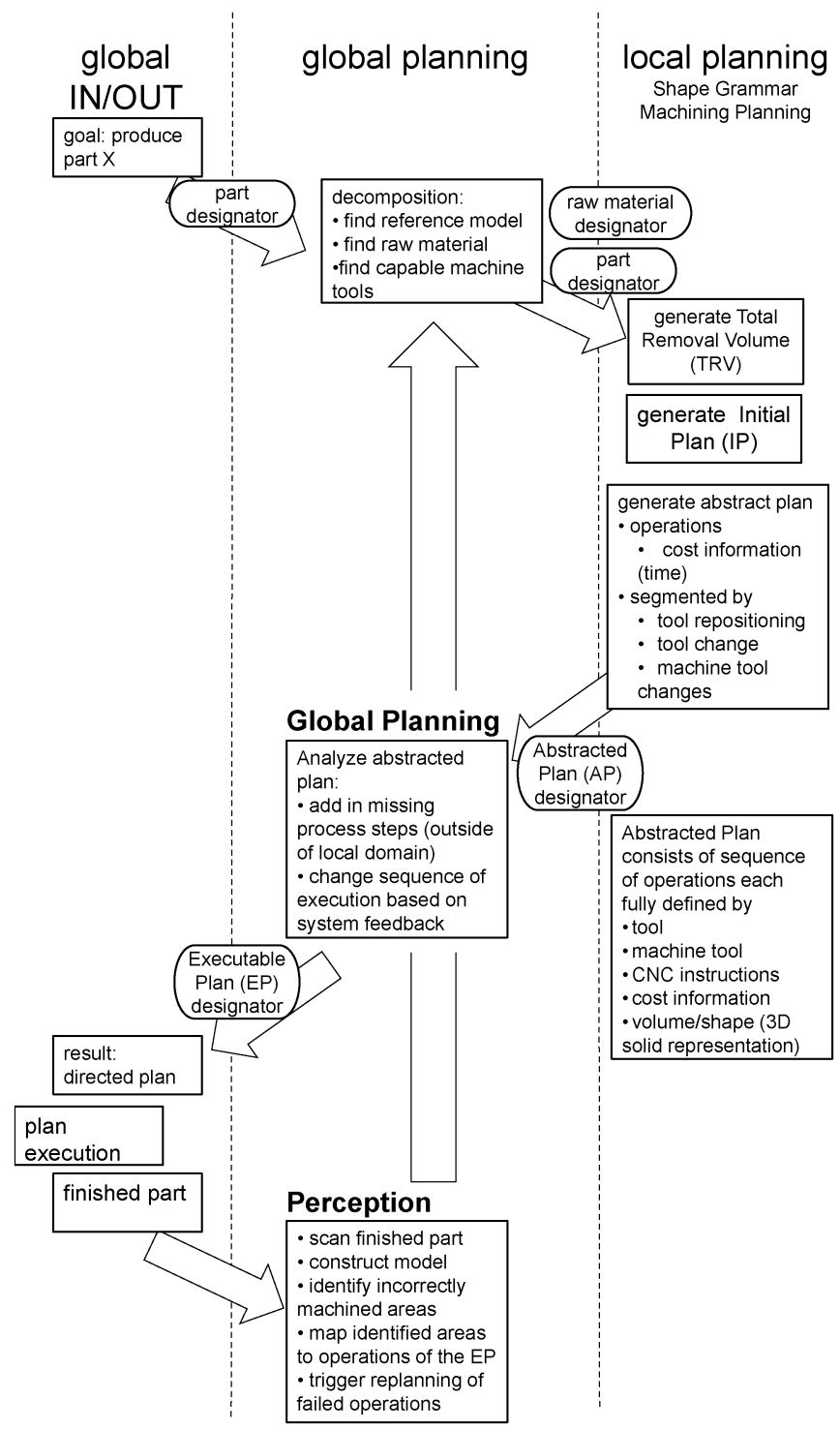

Fig. 12. Process for the integration of local and global planning and perception for part manufacture.

all necessary machines of the manufacturing system. When the manufacture of the part is finished, it can be inspected using the described perception techniques. Faulty areas of the part are identified and mapped to operations of the EP. This is possible since the EP contains the expected resulting shape of the part after every operation. The incorrectly executed operations can then be replanned using the original part models from the EP in combination with the reference representation of the part (i.e., reference edges or reference PC) avoiding resources involved in the incorrect operation. Through this, a faulty part can still be remachined to achieve a correct result autonomously. Additionally, the image of the current scanned part and the derived shape can be stored in the knowledge base for future reference regarding failure analysis. Overall, the specialized local planning system for machining can be effectively integrated with a general, global planning system and thus be integrated in the overall system. The ability to integrate perception into the operation of the system also demonstrated the potential for this combined bottom-up (local planning) and top-down 


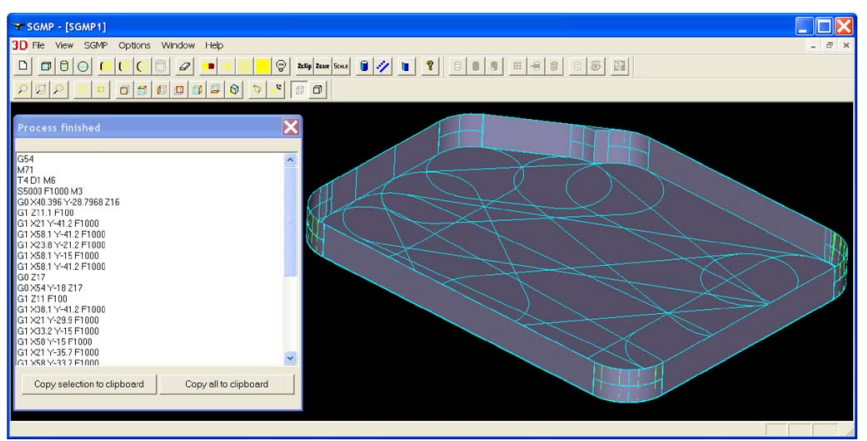

Fig. 13. Screenshot of the machining planning application after plan generation, decomposing the TRV into operations.

(global planning) approach, allowing planning and perception to closely interact.

4) Results: In contrast to traditional tool-path planning where a set of defined features or shapes is recognized from the TRV and then further mapped to fabrication processes, using specific strategies, e.g., equidistant offsetting of the contour and connecting the paths, the system presented in this paper is aimed at being able to deal with a wider variety of parts and fabrication processes. Since the system does not depend on a set of feasible manufacturing features, no maintenance and continuous updating of this set is required, and thus this administration task is minimized. This is also addressed by the ability of the system to generate knowledge models of its capabilities, i.e., the shapes it can remove, from the tool-shape and the machine tool kinematics, enabling the system to adapt itself to new machine tools and tool capabilities. With the method presented, a fabrication system will be enabled to react to missing (raw-) material or machine capabilities, respond to new and changed tools and to replan after machine interruption. The implementation under development of the method using the OpenCASCADE 3D CAD kernel [91] shows the general feasibility of the approach using a single machine tool within a single setup. Fig. 13 shows a screenshot of the planning application developed after the generation of a machining plan. The CNC code displayed on the left side of the figure was generated simultaneously to the application of rules and RVs to the TRV. In the main window, the finished shape of the TRV can be inspected. Currently the part design needs to be constrained to prismatic milling parts to allow for a successful plan generation. The implementation uses a combination of pattern search [92] and Simulated Annealing [93] for search during plan generation. However, different heuristic search methods are currently under investigation to benefit from faster plan generation and improved plan quality in terms of time and possible part variety.

\section{B. Fixture Design and Reconfiguration}

1) Motivation: To enable the efficient and flexible manufacturing of customized products, an approach to the autonomous fabrication [94] and fabrication planning [78] of individual parts on CNC machines, as described in Section IV-A, has been developed. However, insufficient flexibility on the hardware level currently prevents the successful application of these approaches in a manufacturing system. One of the key issues in hardware flexibility is fixture design and fixture device adaption [95] to allow for the secure fixation of arbitrarily shaped workpieces for machining and assembly. To make use of the aforementioned approaches, the hardware system, especially the fixtures, need to be autonomously adaptable and reconfigurable.

2) State-of-the-Art and Research Gaps: Fixture design and fixture automation have been researched for many years. A general overview can be found in the eight survey papers listed by Bi et al. [95]. Still, in the field of automating the fixture process, only a handful of approaches are commonly used in industry nowadays. Among these are the use of pneumatic, hydraulic or electrically actuated elements for locating and clamping the workpiece, as found in active vices or clamps. Normally, these fixtures only automate the clamping process itself and are either not capable of adapting to different shapes of workpieces or need manual interaction to do so. To address the issue of fixing arbitrarily shaped parts, several approaches exist. These include modular fixtures, electromagnetic fixtures [96] and fixtures using phase-changing materials such as magneto- or electrorheological fluids [97], [98]. Further, there are adaptable fixture devices such as the 3-D gripper tool described by Brown et al. [99] and self-adapting fixtures such as vices with movable jaws [100] or pin-array type jaws [101]. Although all of these systems enhance the fixture flexibility, they are limited in their range of use. Magnetic fixtures only work for ferromagnetic parts with at least partially planar surfaces to allow for secure magnetic fixation. The embedding of parts in phase-changing materials is complex and the positioning of the workpiece is not exactly reproducable. Therefore, time consuming position referencing is necessary for each workpiece. The mentioned 3D gripper tool only works for a limited range of workpiece geometries and needs to be reconfigured manually. Self-adapting vice jaws eliminate this need. Pin-array type jaws, based on a matrix of small individually driven hydraulic or pneumatic pistons that, when activated, cast a mold around the parts surface, allow for a great geometric adaptability but again raise the issue of position referencing. The jaws described in [100] consist of two rotatable half-cylinders per jaw. Through rotation, the jaws can adapt to different part geometries. Using sensors to detect the rotating angle for each half cylinder, the exact workpiece position can be computed, so that no referencing is needed. Unfortunately, the underlying algorithm does not work for symmetrical or quasi-symmetrical parts, making human supervision of the process necessary.

The most common systems in industry are modular fixtures consisting of single elements for supporting, locating and clamping the workpiece, that can be mounted on a table with a dowel pin-, hole- or T-slot pattern. A human expert designs and assembles each fixture using these elements. Using a zero-point locating system, the tables, with preassembled fixtures and fixed workpieces, can then be passed around in a manufacturing system and be fixed for machining. Although these systems offer a great adaptability through reconfiguration of the whole fixture, they are very limited regarding their degree of automation. The design and assembly of each fixture is very time-consuming as it has to be carried out manually by the fixture expert. Approaches to automate the assembly of modular 


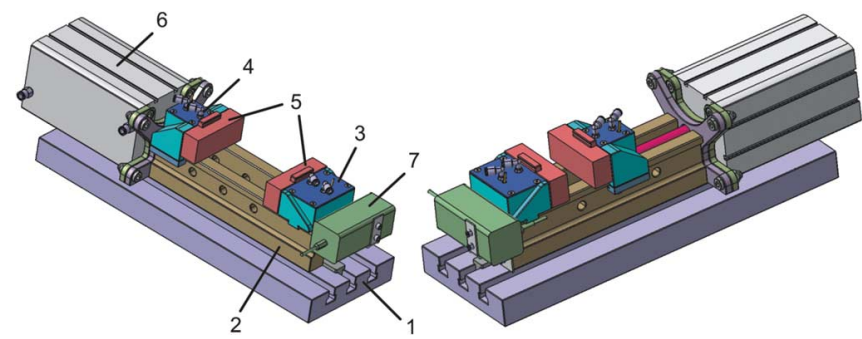

Fig. 14. Structure of the reconfigurable vice.

fixtures using robots are very complicated and expensive [102] and no industrial application is known.

In the field of automating the fixture design process, several methods exist. One possibility is using an ontology to represent fixture knowledge in combination with an agent-based system [103]. Pehlivan and Summers [104] and Cecil [105] give an overview of the work in Computer-Aided Fixture Design (CAFD). Although the use of these methods increased in the last decade, they generally only address the issue of supporting a human expert in designing fixtures. They do not address the issues of autonomous fixture design or automatic reconfiguration and reassembly of the fixtures. As none of the existing solutions fully covers the requirements of a cognitive factory, a new flexible fixture device is under development.

3) Approach: The goal of the approach was the development of a flexible fixture device for the CNC milling machine within a given FMS. The development was carried out systematically according to carefully defined requirements [106]. Some of the key requirements are that: 1) the device can be reconfigured and adapted solely using the existing devices within the manufacturing system (i.e., machine tools, handling robots, storage, and conveyor belt) and 2) similar clamping forces (max.+ $5 \mathrm{kN}$ ), a similar clamping width $(0-130 \mathrm{~mm})$ and a similar positioning precision $(+-0.01 \mathrm{~mm})$, regarding the existing fixture, can be achieved. More than 100 functional solutions were created, evaluated and revised, using classical methods of product development, until the final solution was derived.

4) Results: The result is a fully automatic, reconfigurable and adaptable pneumatic vice. To reconfigure the vice, the clamping jaws can be exchanged by the existing handling robot, without the need to recalibrate the machine tool. To adapt the vice to new part geometries, the jaws can be machined by the machine tool on which the fixture device is installed. The overall design of the flexible vice is shown in Fig. 14. The vice rail (2) is mounted to the machine table (1). The vice's anvil block (3) is mounted to the rail opposite to the movable slide (4). The exchangeable jaws (5) are held in place by pneumatic pistons mounted on the anvil (3) and the slide (4). The slide (4) is driven by a pneumatic piston (6). A cable actuated position sensor (7) is used to control the clamping width of the vice and the correct fixture of a part by variance comparison. Additional noncontact sensors detect the correct locking and unlocking of the jaws.

Since the jaws have a similar weight to the workpieces, the same robot used to insert workpieces into the machine tool can be used to exchange the jaws. This implies that the robot can be smaller, and therefore cheaper, than a robot capable of exchanging the complete fixture device, as required by current

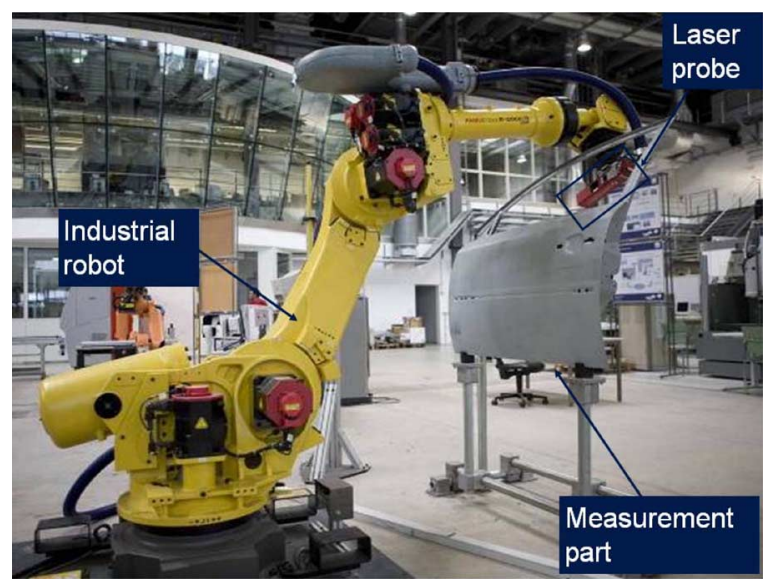

Fig. 15. Robot-guided optical scanning devices.

zero-point fixture systems. Using pneumatic elements to (un-) lock the jaws and the handling robots to exchange them, the reconfiguration can be achieved autonomously without human intervention. The jaws can be exactly repositioned in the vice after the exchange, which makes it unnecessary to recalibrate the origin of the machine tool in order to produce the parts with sufficient precision.

If no fitting jaws for a certain part exist, a set of blank jaws can be machined to the desired shape on the machine tools. To create the design of the jaws, a knowledge-based approach is being developed, able to synthesize valid jaw designs for a given part, based on the 3D model of the workpiece. A key challenge in this process is to find an optimal orientation of the part within the fixture. Liu [107] and Zhu [108] show different approaches to overcome this challenge from the field of fixture layout or robot grasping. Once the jaw design is created, the $\mathrm{NC}$ program for their machining is derived using the method for autonomous machining planning [78] described in Section IV-A. This enables the autonomous adaption of the fixture and therefore the fixation and fabrication of a wide variety of part shapes.

According to the requirements, the current device is limited regarding the maximum part size and clamping forces, mainly due to the use of pneumatic pistons to create the necessary forces. This solution is chosen out of weight and cost considerations. However, using hydraulic pistons, the vice can be easily extended to higher clamping forces and larger part dimensions, thus making it more applicable in industry. The underlying principle of the flexible fixture device can also be extended to grippers for the handling robots, adding further flexibility on the hardware level. The ability to reconfigure the fixtures fast and flexibly minimizes change-over time, while increasing production time. For handling, transportation and installation of the jaws, the existing control and planning systems can be used, integrating the jaws and their configuration into the manufacturing system and its existing processes. Overall, the approach is an important enabler of flexibility, especially supporting the autonomous operation in a cognitive factory for the fabrication of customized and engineered-to-order parts. In addition to the development of an autonomous part orientation and jaw design method, current work also includes creating a hardware prototype of the fixture device and integrating it into the manufacturing system for further validation. 


\section{Robot-Guided Quality Inspection}

1) Motivation: In dimensional quality inspection, acquiring the shape data of the physical objects to be inspected is essential [109]. Robot-guided optical scanning devices (compare 15), can be deployed for the flexible digitalization of nearly all kinds of free-form surfaces that require high-resolution sampling [110]. Despite this enormous advantage, the wider implementation of such systems, especially in the case of small lot sizes or customized parts, is still far from being reached. This is partially due to the extensive amount of time and human effort required to manually program the robot paths for every new part using trial-and-error approaches [111], [112]. Since the laser scanner trajectories have to simultaneously satisfy several constraints [111], [113] using tedious and time consuming manual teaching methods often result in, over- and underscanning or missing of some interesting filigree regions [112]. Therefore, inspection path planning is an important, complex part of robot-assisted inspection tasks and needs to be automated in order to overcome these challenges [114]-[116].

2) State-of-the-Art and Research Gaps: So far, essentially two distinct approaches which mainly differ in the amount of planning done online and the amount of a priori knowledge about the part to be digitalized, have been investigated. Online approaches without a priori object shape knowledge [117], [118] assume that the artifact to be scanned is enclosed in a bounding volume. According to that assumption simple grids of regularly spaced robot poses are generated orthogonal to the sides of the bounding volume.

The main drawback of this approach is that the generated path cannot maintain a regular pattern of line stripes to reconstruct quality surfaces out of the point cloud. Furthermore, a lot of unsampled regions are spawned when digitalizing complex parts. This makes the gathered point cloud only adequate for reverse engineering purposes unless it is complemented by driving the robot manually around the unsampled areas. Offline approaches with a priori object shape knowledge were successfully conducted by many researchers. The methods are basically built around the visibility or accessibility theory [112], [114]. The algorithms introduced in those works use visibility or accessibility cones to compute a set of points from which the scanning devices can freely access the part by satisfying a set of constraints.

Additional reported methods are based on slicing algorithms to divide a CAD model into a number of sections in such a way that each section can be covered by the laser stripe width [119]. However, it is unclear where the slices should be spotted in order to obtain good results.

Most of the outlined methods and systems address the scan planning issue as an isolated problem without incorporating the topology of the scanning scene. The yielded scanning paths lie in a meander- or zigzag-fashion over the surface containing the features to be collected.

Since the implemented systems are based on coordinate measuring machine (CMM), respectively, Cartesian robot-based, kinodynamic constraints play a minor role or are simply not considered. Only the collision between scanner and part is taken into account. Moreover, no methodology is introduced to automatically capture specific curved regions of interest such as edges, corners, slots, pins, without scanning at least a large portion of the part. Those methods and system cannot also cope when an active real-time control of the sensing process is required to resolve mismatch between the virtual and real world, hence, assure that the robot stays on the right track.

3) Approach: Consequently, the novel approach proposed by the authors tackles the four main bottlenecks while carrying out automated inspection tasks: the optimal positioning of the workpiece, the selection of the features to be gauged, the automated generation of optimal scanning paths and corresponding smoothly shaped robot movements and the real-time control of the scanning process. This new approach rests on the observation that artifacts such as edges, corners, slots and pins are critical for manufacturing height quality grade parts. Thus, using the spatial information of the workpiece in the form of a CAD model, the part feature line contours are automatically extracted as given by the design-engineer. The user then decides which of the extracted features are relevant for the inspection task and optimal scanner poses as well as collision free robot motion sequences are automatically generated. The following are major steps of the methodology.

Features contour lines extraction and inspection task description: The aim of this step is to isolate features contained in the CAD model. Given tessellated, structured surface model of parts, feature regions distinguish themselves by high curvature, respectively, heavy direction change of surface normals. Hence, the extraction of features is achieved by tracking the later across the meshed model. For this purpose, image fairing techniques described in [120] were adapted. The developed algorithm is based on a three-stage calculation scheme including the following.

A Preprocessor stage in which the input meshes are subjected to a filtering algorithm in order to heal the model from errors, refine the initial mesh, eliminate the redundant data, establish vertex connectivity in triangle fashion and finally store the processed data in a proper structure for further use.

A Classification and selection stage in which a Second-Order Difference (SOD) classification operator as presented in [120] is used to assign an importance factor to every edge of the polygonal mesh. The importance factor adopted here is proportional to the dihedral angle between the two polygon faces that enclose the edges. Afterwards, the model is decimated using a hysteresis threshold algorithm, which filters the most nonsalient regions out of the mesh and simultaneously indentifies the edges belonging to a feature contour or to its neighborhood.

Finally, a Skeletonizing and pruning stage where using the medial axis transformation operator [121], a skeletal remnant of the output from precedent is produced in form of a collection of piecewise linear curves describing the salient features of the mesh. Fig. 16 illustrates the result of the algorithms applied to the CAD model of a car side panel. The inspection task can be further specified manually by selecting a subset of desired feature contours out of the previous stage results (compare the highlighted features in Fig. 16). The complete feature set can also be automatically considered if no human interaction is desired.

Tool path planner: Next, for a given point of the feature line, the Tool Path Planner generates a set of robot-independent scanner poses (spatial placement and orientation) that ensure a robust coverage of the feature. Robust coverage implies that the 


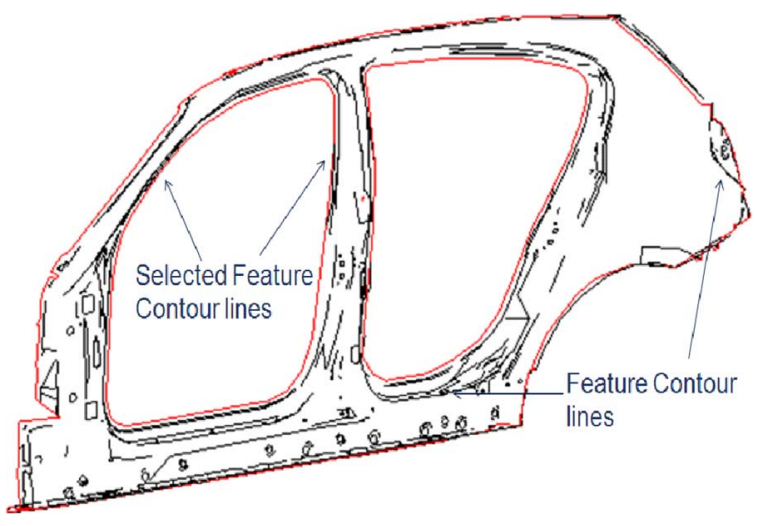

Fig. 16. Feature lines of a car side panel (The highlighted ones chose manually selected contour lines.)
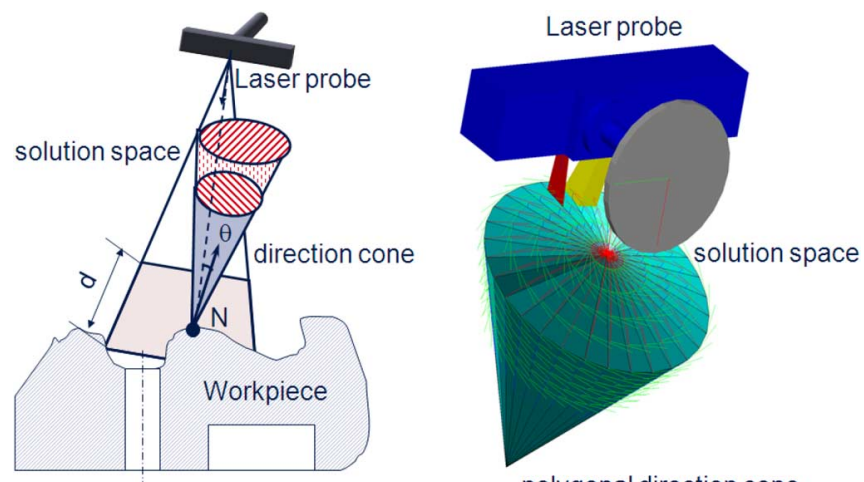

polygonal direction cone

Fig. 17. Geometrical constraints while scanning.

following constraints illustrated in Fig. 17 [111]-[113] must be satisfied:

1) View angle: The angle between the incident beam and the surface normal of each point of the laser stripe should be less than a given limit angle. Ideally, this angle should amount to zero degrees.

2) Field-of-View (FOV): Any measured point should be within the length of the laser stripe $B$.

3) Depth-of-View (DOV): Scanned lines must be within a specified range of distance $d$ from the laser source.

4) Self-occlusion: The incident beam as well as the reflected beam must not interfere with the workpiece.

5) Clearance for the laser probe: The probe has to be collision-free with the workpiece.

To fulfill these requirements, the decoupled approach implemented in this work can be summarized as follows: First, for each knot of the feature line, a polygonal direction cone aligned with the normal vector of the knot is calculated, considering the generalized laser scanner model plus constraint one and two. Afterwards, the self occlusion test is performed heuristically by tilting or scaling down the direction cone without violating the first two constraints. Simultaneously, the workpiece, the cones and the laser probe are checked for collision. For this purpose, a scalable CAD model of a cone as well as the CAD models of the part and the laser probe, are passed to the Planner. Collision monitoring is carried out by the third party Proximity Query software Package (PQP) [122]. Once the direction cone at each point of the feature

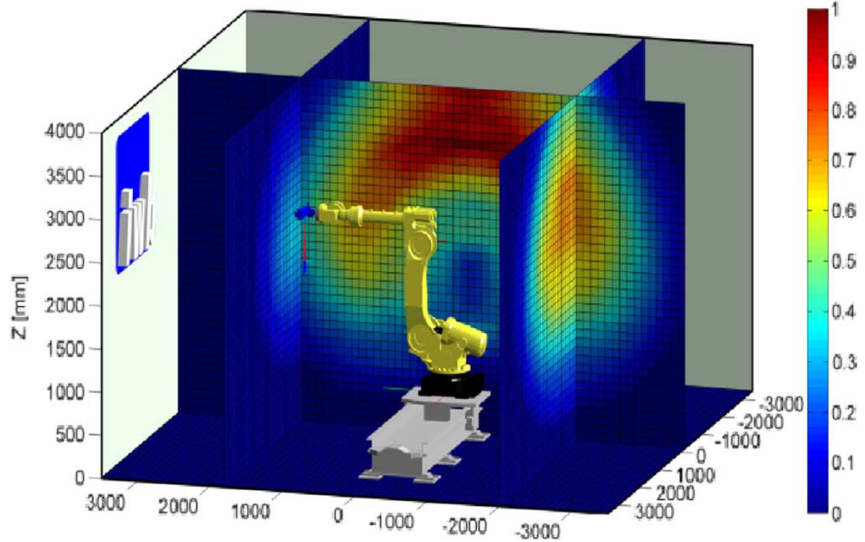

Fig. 18. Scannability map of a linear track-mounted robot-based 3D scanning system. The result was save in a 3D matrix and sliced for better visualization.

line is computed, an area that constitutes the solution space for the collision free positioning of the laser probe is isolated on its upper part using constraint three (compare Fig. 17).

Robot motion planner: Before addressing the issue of motion planning, the positioning of the workpiece in the manipulator's workspace needs to be considered, since its can noticeably influence the path planner. Hence, it will be a great benefit if the chosen workpiece posture lies in an area where the robot posses outstanding dexterity index values. The dexterity index is computed via the manipulator Jacobian matrix and measure how far a robot is from kinematic singularity at or near given targets. This index also quantifies the robot's ease of arbitrarily changing position and the orientation of its tool center point at a given posture [123]. Accordingly, we introduce the new notion of a robot scannability map with manipulator-guided scanning systems. The map should give a graphical description of the scanning system's ability to digitalize a part within its working area. It should also provide information to all robot motion planner so they can avoid singularities, as well as configurations subjected to collision. The scannability map shown in Fig. 18 was obtained by performing a kinematics workspace analysis similar to [123]. The red fields denote the region where the workpiece should be positioned for optimal access by the laser scanner, where dark blue indicates areas with bad scannability attributes.

The Robot Motion Planer itself has the task of planning the trajectory of the robot manipulator's joints based on the previous computed tool solution space. Its core consists of five algorithms. The first algorithm computes a preliminary feasible path consisting of a sequence of viable scanner poses along the targeted feature contours. This is done by heuristically searching for a scanner pose residing in the solution space at each cone and connecting them with piecewise linear segment lines. Thereby, the best next heuristic algorithms used here, try to keep the sensing direction as perpendicular as possible to the sensed point. The second algorithm presmoothes the obtained path using an elastic-band-based algorithm. The third Algorithm interpolates the path using cubic splines to form smoothed robot joint's curve and computes afterwards the inverse kinematics solutions for each intermediate point of the interpolated path. The robot configuration yielded by 

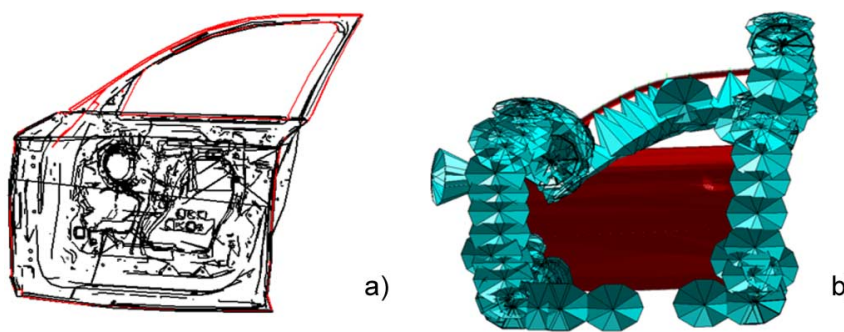

b)
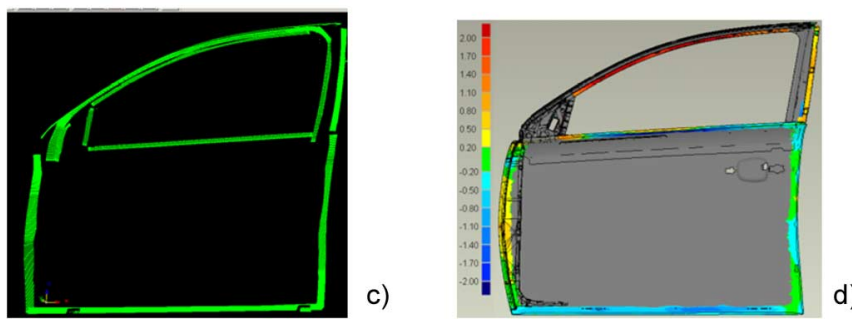

Fig. 19. Experimental result: (a) extracted and selected feature lines; (b) solution space; (c) point cloud; (d) shape error map.

the inverse kinematic are then checked for singularity and collision. By positive hits, the algorithm performs minimal modifications on the Cartesian pose of the laser scanner pose to remove the handicap. The same procedure is subsequently conducted at each intermediate path point until an adequate robot configuration is retrieved. The fourth algorithm is based on the Probabilistic Roadmap Planner developed in [124] and inserts collision free intermediate poses for transfer motion so that the complete motion of the robot is fully determined. To achieve high-level process safety, the Robot Motion Planner explicitly takes the topology of the current robot workspace into account. In cases where multiple robots are needed to achieve an inspection task, the laser scanner path is first partitioned and assigned to the individual robots, taking each of their physical limitations into account. The fifth and last Algorithm takes action at the phase of the system operation. It consists of a PI feedback controller, which monitors the scanning data in real-time and tries to keep the surface being digitalized within the Laser scanner's depth of view. Hence, this Algorithm ensures that the robot stay on the right track, even if the virtual robot cell where the path planning is carried out, marginally differs from the real robot cell.

4) Results: A prototype system that utilizes the described methods and algorithms has been implemented using Matlab and $\mathrm{C} / \mathrm{C}++$. To evaluate the performance and robustness of the implemented algorithms, experiments were carried out on a car door. The equipment used includes a six-axis robot, 3D laser scanning system. Fig. 19(a) shows the extracted contour feature lines of the car door. In Fig. 19(b) the solution space along the features lines are depicted. Fig. 19(c), respectively, Fig. 19(d) shows the collected point cloud and the shape error between the point cloud and the CAD model.

\section{Hybrid And MANUAl PRocesses AND Operations}

\section{A. Human-Robot Cooperation}

1) Motivation: In general, issues concerning the cooperation between humans and industrial robots are emerging more and more in ongoing research fields, focusing on human machine interaction, as well as industrial safety requirements, issues, and union decrees. Humans and robots must share certain knowledge about the task to solve, about each other, and about external constraints, including the environment, in order to cooperate effectively. Today's industrial robots have no ability to "see" humans as cooperation partners, because they cannot perceive their surroundings at all. According to the research results of cognitive neuroscientists, a robot must engage in joint action: joint attention, action observation, task sharing, and action coordination in order to achieve successful joint action [125].

Joint Attention: For cooperation between robots and humans, it is important that both partners coincide on the same objects and topics and create a perceptual common ground and shared representation of reality [125]. That means that the system has to be able to know where the human's focus-of-attention is. The focus-of-attention can be extracted from data gained by visual sensor devices (cameras) using tracking techniques (e.g., [126]) or from data gloves [127]. Therefore, the system needs to recognize, for example, a pointing gesture, compute the location indicated by the human worker, and then transform this information to estimate his focus-of-attention. Thus, an internal system representation of the environment that is always kept up-todate with information from the sensors (workspace model) is required.

In addition to pointing gestures, the human worker's head orientation can be used to determine his focus-of-attention. According to behavioral studies [128], head orientation is directly connected to a human's focus-of-attention. Determining head orientation can be achieved with tracking techniques, using mutual information presented in [129].

Joint attention also implies that both partners coincide on the same objects. In a production scenario, this includes objects like workpieces and tools that might be used.

Task Sharing: To manage a predefined goal, in this case the assembly of a product, a robot-system needs to know about the task in the production process, the human worker, and the necessary skill rated steps. Therefore, the representation of the tasks should be as generic as possible, so that role allocation can be changed dynamically, even during production.

Action Observation and Coordination: All information perceived by the sensors builds up an internal representation of the working environment, the workspace model. In addition to the perceived data, the workspace model can also carry information about the inventory (e.g., how many parts are stocked), an assembly line (e.g., which part is on the assembly line), and the worktable (e.g., where parts that are already in use can be placed).

This information is used in the decision making process of deciding whether to infer in the next action step, along with the knowledge about the task provided by the current building plan, sensor information, and extracted features (e.g., the human's focus-of-attention). The system has to begin executing the likeliest next step in order to reach an anticipatory behavior that is important for efficient collaboration. If new sensor-input changes the situation and thus the decision, the system must change its behavior seamlessly.

To control the movement of the robot (Motion Control), real time control of the robot is needed. Because the robot and the 
human worker share the same work space, the movements of the robot cannot be programmed offline for safety reasons. After starting to execute the next step towards reaching the assembly goal, the robot has to be aware of obstacles (e.g., the human worker's hand, body, or arm) and react in real-time to avoid collisions with them.

Assembly modules of today's factories can be subdivided into the following three classes: fully automated assembly, fully manual assembly, and a combination of manual and automated elements (the so-called hybrid assembly).

2) State-of-the-Art and Research Gaps: The state-of-the-art in human-robot collaboration is mainly based on a master-slave level, where the human worker teleoperates the robot or programs it offline, allowing the robot to execute only static tasks. To ensure safety, the workspaces of humans and robots are strictly separated either in time or in space. For instance, human workers in the automobile industry are completely excluded from the production lines, where automated robots execute assembly steps. On the other hand, robots are not integrated in assembly line manufacturing along with human workers. These approaches do not take advantage of the potential for humans and robots to work together as a team, where each member has the potential to actively assume control and contribute towards solving a given task based on their capabilities. Such a mixed-initiative system supports a spectrum of control levels, allowing the human and robot to support each other in different ways, as needs and capabilities change throughout a task. With the subsequent flexibility and adaptability of a human-robot collaboration team, production scenarios in permanently changing environments, as well as the manufacturing of highly customized products, are possible. Future assembly systems could be characterized by a direct cooperation between humans and robots without the use of separating safety equipment or safety areas. The fundamentals for this cooperation are currently being created from a technical and legal perspective. For an effective human-robot-collaboration, new safety methods have to be developed and proven. Human workers, as well as objects in the environment, have to be detected; collision avoidance algorithms must ensure the safety of persons and equipment.

3) Approach: The innovation lays in the creation of a smart working environment in which a human and an industrial robot can work together on a peer-to-peer level, overcoming state-ofthe-art techniques to shut the robot down if a human comes close in order to maintain a safe working environment. This is achieved by the use of multiple modalities to analyze current actions, to reach pro activity (anticipatory behavior), and to ensure safety for the human co-worker (reactive behavior).

Therefore, a special hybrid assembly cell, which can be seen in Fig. 20, is integrated as a workstation in a Cognitive Factory research environment [130]. The hybrid assembly cell primarily consists of an industrial robotic manipulator, a shared workbench, where the Human-Robot interaction takes place, a tool change station for the manipulator, and sensors to monitor the ongoing activities within the cell. To connect this hybrid assembly station to the remaining manufacturing units (fully automated assembly, purely manual assembly, automated storage rack), a conveyor belt is located directly behind the

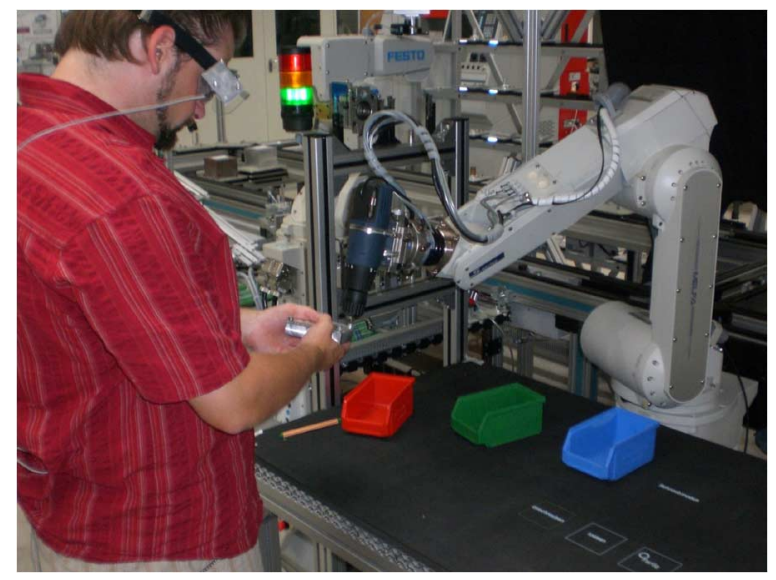

Fig. 20. Picture of a worker wearing eye-tracking glasses and fixing screws together with the robot on a product in the hybrid assembly cell (CoTeSys/Kurt Fuchs).

workbench to exchange parts and other products. The following explains the specification of this setup in greater detail. The general idea behind the hybrid assembly is to construct a workpiece by exploiting the advantages of the cooperation between a human worker and an industrial robot. Thereby, the cognitive skills and dexterous manipulation capabilities of the human are combined with the precision, repeatability, and strength of the robotic counterpart, which enhances the current productivity in general and decreases the physical efforts of the human. Therefore, a sample product was selected, including tasks appropriate for the human worker (wiring), dedicated for the robot (glue parts together), and for both of them (mounting and fixing screws). The conveyor belt access was managed autonomously by the robot to exculpate the human from this task. The robot also hands over the required parts when they are needed in the assembly process. Otherwise, these parts are stored in a local buffer, to be used later on. The hybrid assembly cell is equipped with a Mitsubishi RV-6SL manipulator arm, which has a maximum payload of six kilograms, six degrees of freedom in motion, and a spherical workspace with a radius of 0.902 meters. Furthermore, the robot controller allows online programming if the position data is updated within a timespan of seven milliseconds. Mounted on its tool center point, a tool-change unit provides desired flexibility in handling operations. Therefore, several different kinds of grippers (two parallel grippers and one centric gripper) and end effectors (screw driver, observation camera, glue-unit) are within the robot's workspace and can be exchanged, depending on the next steps of the current procedure, without manual interference. The intelligent controller is aware of what tools are needed to perform specific tasks, based on the production plan. For example, if the system requests gripping an object and the tool currently installed is not appropriate for this handling operation, the controller changes autonomously towards the correct end effector or gripper. This operation happens without the human having to implement the necessary commands explicitly, making the planning of the task less complex. Furthermore, a force torque sensor delivers data about occurring forces, which 

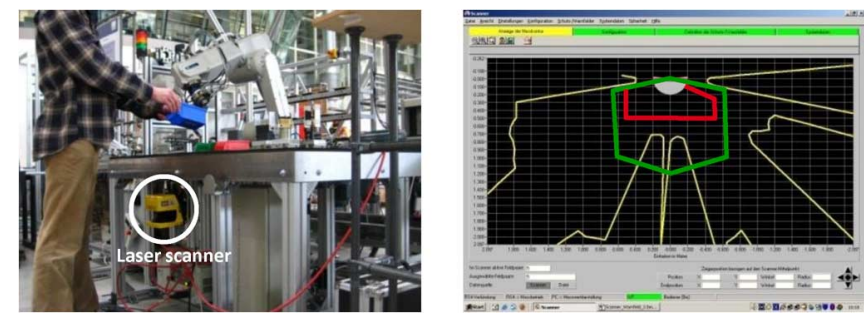

Fig. 21. Left: laser scanner-experimental setup; right: laser scanner-data evaluation.

can be used as valuable information for interaction situations or manufacturing processes.

A camera is mounted above the workbench and provides a view of the entire area of interaction. Information about rotation and location of objects in the scene and the positions of the worker's hands are extracted from this image source as well. For image segmentation and increased safety within the interaction process, the depth data delivered by a Photonic Mixer Device (PMD) camera is used together with information about the robot's current position. A further camera is applied to gather information about the head and face of the worker. A desktop array microphone used for video conferencing is used to capture the audio signals of the worker, required for speech recognition. A haptic input modality to the system is provided by buttons, which are projected onto the workbench with a table projection unit. This projection unit is also used to provide the next assembly instructions in an ergonomic way. However, an additional input channel was setup by introducing eye-tracking glasses, as presented and discussed in [131], into this working environment. This allows the human worker to enable system functionalities by the use of gaze information or to select options by simply looking at the buttons.

Safety Sensors: For interactions between humans and industrial robots, the position and orientation of both entities must be known to avoid any collisions between human worker and robot.

To detect the human, a laser scanner is used. The scanner is mounted below the working table (see Fig. 21). Due to safety reasons, the position data of the human has to be verified by a second redundant system. In case of data loss of one system the second system can release an emergency stop command. A matrix of safety shutdown mats can evaluate the data of the laser scanner to verify the standing position of the human worker. Therefore, a matrix of safety shutdown mats on the floor can detect where a human is standing from the pressure of his feet [132].

System Controller: In a first phase, the control of the HumanRobot interaction system, presented in [133], has been realized with a finite state machine to build up an efficiently robotic work cell. During the first evaluation, we found, that this kind of system controller did not meet the requirements of flexibility. Therefore, the controlling unit was replaced with an expert system based on first-order logic. This expert system uses the Real-Time Data Base (RTDB) Control-Object to exploit the self-reporting mechanisms of these intelligent software modules. The skills of the available and running modules, i.e., the
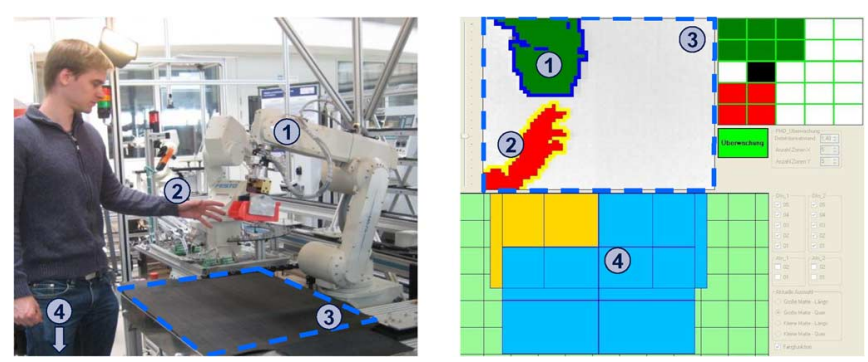

Fig. 22. Left: real production cell; right: abstraction on system level.

input and output modules, can be enumerated and used for autonomous planning purposes considering specific constraints, such as computational power or inter module dependencies, etc.

User Adaptation: Adaptation on multiple levels will increase the performance of the system and also the user's acceptance. For example, the skin color model for gesture recognition can be learned based on the color histogram of the facial region, as presented in [134]. Additionally, the language model can be adjusted according to its present user, to provide a natural language speech input to the system. Further preferences, like handover positions, preferred interaction modalities, and distances, etc., can be learned by the system and stored in this user model.

4) Results: Some safety aspects are shown in Fig. 22 (left: real production cell; right: abstraction on system level). For the detection of humans, five procedures can be used.

With a matrix of safety shutdown mats the rough position of humans can be seized. This can be verified with a laser range scanner. With marker-based object-tracking and vision-based tracking algorithms, the head and hands of a human worker can be localized very precisely. Observation of the working table is realized with a PMD camera.

Within the project, interaction between robots and humans in a common work space was examined on a research platform. Here, the individual strengths of the interaction partners are linked. The robot is used for the handling of heavy construction units and for the execution of repetitive tasks like screwing. Humans are used to implement sensitive and fine-motor work, like fixing a cable in cooperation with the robot. The cooperative efforts must be guaranteed by the integration of different safety components in the system and an intelligent path planning method so that the robot is closely integrated in the assembly process and can optimally adapt to the actions of humans. This way, humans can interact and cooperate, using their superior cognitive developments and high fine-motor abilities with robots close by with the help of multimodal interfaces.

\section{B. Quality Assurance and Process Control in Laser Welding}

1) Motivation: Most robots functioning today are used in industrial production, so the potential for cognitive capabilities is high in this area [135]. In manufacturing, certain production processes are especially hard to control and observe in regard to quality; material processing with laser beams is one of these. The strong radiation and process emissions that result from working with high powered lasers require specialized sensors and excellent controlling techniques. Therefore, the aim of this research is to improve the quality assurance and process 

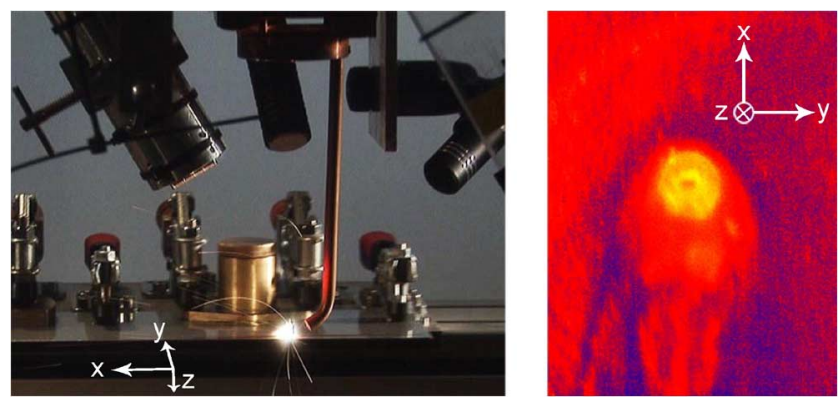

Fig. 23. Laser welding process equipped with acoustic sensors; in-process image taken with coaxial camera.

control in laser material processing with artificial cognitive capabilities, such as learning how to weld or cut metals. Laser beam welding or cutting experiments, under real industrial conditions, shall then validate the improvements by using technical cognition in production or manufacturing systems.

The control of laser beam welding has challenged research and development teams for decades. Many sensor and quality assurance systems have been invented and introduced to industrial assembly lines, most of which use cameras and photodiodes for monitoring [136], [137]. Systems in industrial use today are set up manually and undergo many trials. Any variation in process parameters often necessitates a recalibration, which might even require the assembly line to be halted. Research has offered promising approaches for controlling laser welding processes [138] and for teaching machines how to handle them [139]-[141]. In a Cognitive Factory, many different sensors are combined and real-time machine-learning techniques to improve process quality and control are developed.

The cognitive capabilities under evaluation can be separated into four categories: first, abstracting relevant information; second, learning from a human expert how to control; third, acting on learned knowledge; and fourth, autonomous decision making in previously unknown situations.

2) State-of-the-Art and Missing Research: state-of-the-art sensors for laser material processing are usually insufficient to monitor process quality online if only one kind of sensor is used. Therefore, different kinds of sensors are utilized to combine many partially weak sensor signals for an improved combination. For the cognitive laser material processing system, we introduced cameras, photodiodes, and sensors for solid-borne and air-borne sound, offering a lot of valuable process information, as demonstrated in Fig. 23. Receiving data from many different sensors is vital to be able to abstract the relevant information while reducing the amount of data in order to process information in a reasonable time. Linear and nonlinear dimensionality reduction enables us to map an high-dimensional observation space $Y$ to a low-dimensional feature space $X$. All of the data from the applied online sensor system can be represented by a 26.603 dimensional vector, which can be reduced to only 33 dimensions, representing the relevant process-information by combining different dimensionality reduction techniques. Thus, we are extracting characteristics that enable the CTS to perceive the real world [142]-[145].

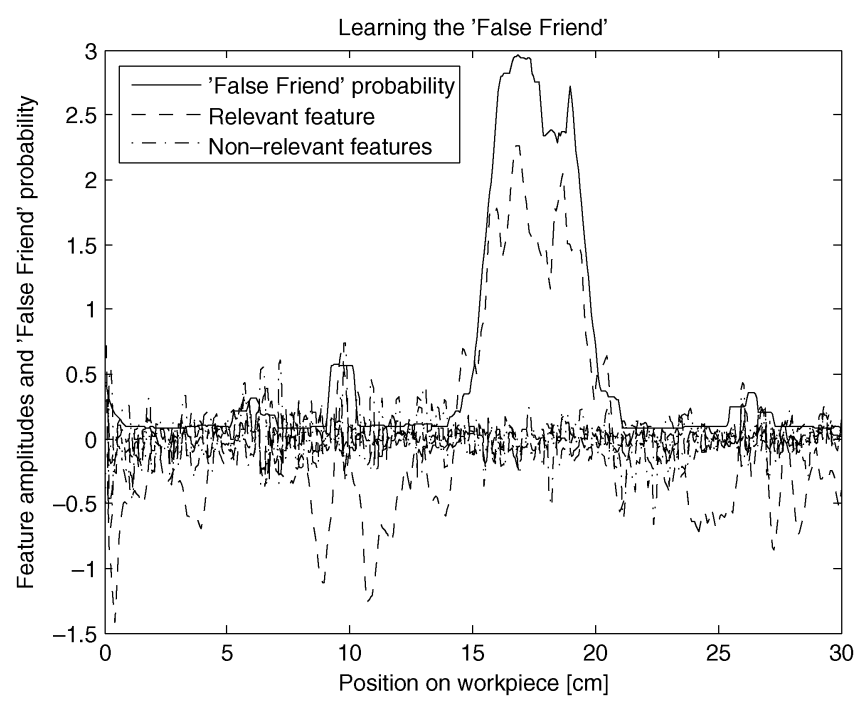

Fig. 24. Features and probability related to the "False Friend" process error.

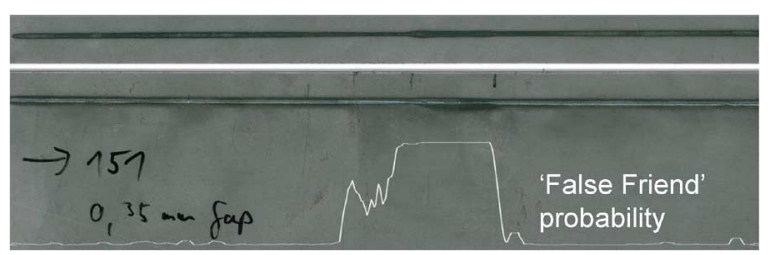

Fig. 25. Probability of the "False Friend" process error for a test sample.

3) Approach: Laser material processing systems are usually set up and processes configured by human experts. In order to simplify and accelerate this process, the cognitive capability for learning is implemented in the cognitive laser processing system. In the learning phase, the system performs a test action such as a laser power ramp for welding or a processing gas ramp for cutting. The human expert points out, over a graphical and acoustic interface displaying the workpiece, how the processing result would be classified for the different workpiece areas, such as a poor weld with not enough laser power, a good weld, and a poor weld with too much laser power applied. Our system stores this information together with the extracted characteristics described above with a classification technique. We evaluate several classification techniques for our purposes, such as Support Vector Machines, Artificial Neural Networks, and Fuzzy k-Nearest Neighbor. All of the named classifiers achieved good results; the extracted characteristics seem to be separable from each other. However, the knowledge learned from the expert makes the system capable of performing improved quality monitoring because a considerable amount of joint sensor data input is used. Even previously hard-to-detect faults can be monitored, such as the so-called false friend, which may occur when the gap between the parts to be joined is too wide, as shown in Figs. 24 and 25. The performance of the monitoring results of our system will be evaluated for further scenarios in the future [135].

4) Results: Having improved monitoring results, different closed-loop control experiments are performed on the basis 


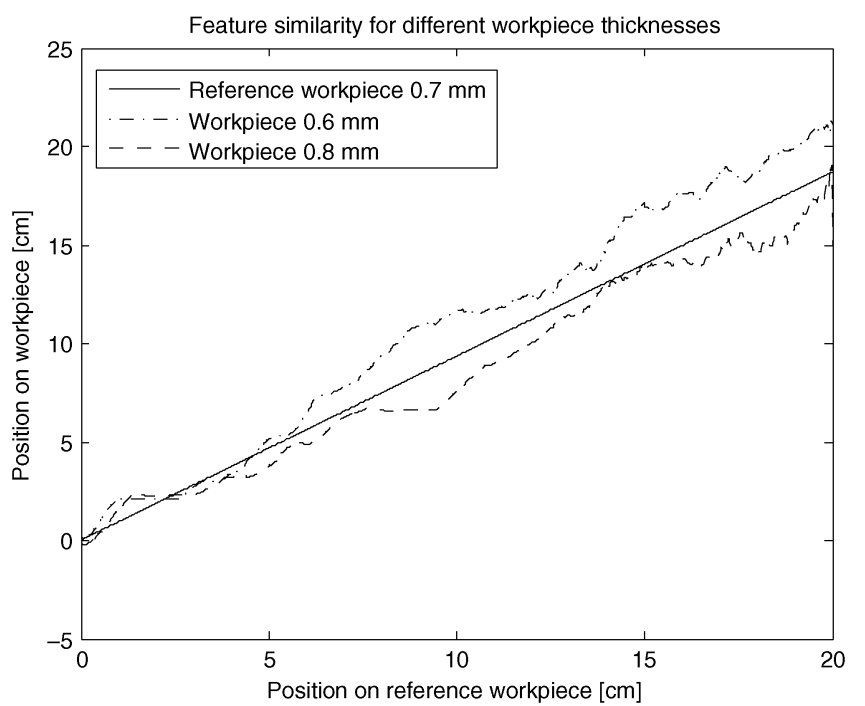

Fig. 26. Similarity of features acquired on workpieces of different thicknesses while performing a laser power ramp.

of the learnt knowledge. Combining PID-control with the classification results enabled us to do "cognitively control" overlap welds of stainless steel workpieces with laser power, compensating for the focus shift in processing optics by varying the $z$ axis, and reducing the influence of the gap between the parts to be joined. Especially for controlling tasks, real-time data processing is important. Using low data volume characteristics to represent the relevant information enabled us to have closed-loop control cycles of $100 \mathrm{~Hz}$. In experiments, the system was capable of adjusting the laser power in overlap welds and achieve a processing result that was previously defined by an expert, thus the machine's capability to learn initially from a human is validated. In other experiments, we successfully measured the sharpness of images. Because the observing camera is integrated into the optics used for processing, it was possible to detect the focus shift, which occurs when the optics heat up because of energy absorption. This and other learned background properties may be used to control the $z$ axis and vary the distance between the workpiece and the processing head. Furthermore, since we were able to detect a false friend, it was possible to reduce its influence by adjusting the laser power. Further experiments can evaluate the limits of the cognitive laser material processing system [142], [146]-[149].

Even though every attempt was made to keep all processing parameters constant for a configured process, influences may occur in varying workpieces, such as changes in mounting and workload. To cope with this challenge, we integrated a learning scheme. A novelty check with the stored knowledge can detect new or previously unknown situations. In this case, the system performs another test action and classifies the new workpiece. This time it does not need to consult a human expert; it can map the gained knowledge onto the new workpiece autonomously and adjust the process control appropriately. We have tested this cognitive capability for varying material thickness of $\pm 10 \%$; the results are demonstrated in Fig. 26. They indicate that the system is capable of making a judgment and adjusting the applied laser power for receiving the desired welds accordingly. Further techniques for this step will be evaluated, such as Q-Learning in order to increase high-level adaptivity [135], [150].

In summary, we were able to demonstrate cognitive capabilities within a laser material processing system managing multiple sensors and actuators. The system has been able to learn from a human expert how to weld or cut materials, make decisions in completely new situations, and improve monitoring and process control. Future research will concentrate on improved learning capabilities for higher adaptivity and for controlling cycles greater than $500 \mathrm{~Hz}$.

\section{Assistance Systems}

1) Motivation: Besides automated and hybrid production processes, especially for complex products, manual assembly by human workers is indispensable [4], [151], [152]. Human workers have fine motor skills and are able to flexibly perform several tasks. Moreover, they easily can switch between various tasks with changing content. Nevertheless, human mental capacities are limited and resources have to be shared and distributed among the relevant subprocesses. Accordingly, in order to optimize ergonomic as well as economic aspects of manufacturing, the worker needs to be assisted during the whole working process [153], [154].

2) State-of-the-Art and Research Gaps: Existing systems for digital assistance in manual assembly, e.g., optical displays at the workplace, are inherently suboptimal, because they do not take into account the dynamics of the production process. Traditional assembly planning is purely deterministic while using predefined assembly orders. Moreover, none of the existing methods allows for the selection of assembly tasks in real-time. The generation of assembly instructions happens without the comprehension of the actual production environment and the physiological or psychological state of the worker [155]. Individual worker internal models of the complex products and unforeseen events of the factory environment (e.g., missing parts) are not taken into account. However, the display of sequential instructions does not offer an increase in productivity beyond a certain degree [16]. Moreover, little situational support and the resulting deterministic guidance leads to a reduced acceptance of such systems by the worker.

3) Approach: A key to solving these discrepancies is seen in the development of an assistive system that delivers adaptive worker guidance. The system integrates factors of the production environment as well as factors regarding the human worker. Accordingly, information and instructions are presented at the right time and the right place with adapted content. In order to fulfill these goals, cognitive methodologies for the generation of assembly tasks are used. Nonlinear assembly sequence graphs are derived in real-time for the generation of adapted instructions. These require environmentally- and situationally dependent triggered paths on state-based graphs [157], [158]. The graph-based structure integrates product states as vertices and assembly operations and their respective complexity as edges [159]. For this purpose, the current product states are continuously monitored. Assembly states are interpolated from peripheral entities of the production environment (e.g., workbench, 
state-based mapping and processing of sequential tasks

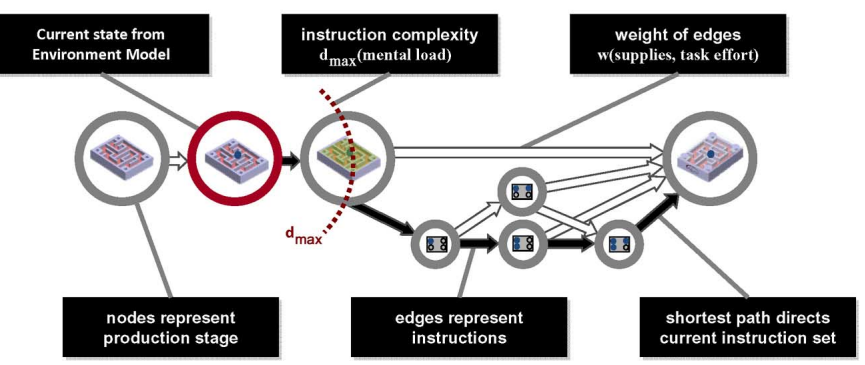

Fig. 27. State-based assembly graph.

storage rack). The adaptation of these graph-based process plans is based on the worker's actions, performance, and mental state. Relevant information about the worker based on implicit worker interaction (e.g., eye and hand movements) and of a database (e.g., worker profile) are merged into a parameter $d_{\max }$ (see Fig. 27). The parameter $d_{\max }$ restricts the maximum step-length on the path of the assembly sequence towards the target of the product, i.e., the degree of detail and complexity of the task displayed to the worker. In this context, a multidimensional task complexity metric is defined while modifying basic execution times through parameters for cognitive task processing of the worker (e.g., perception and selection processes) and product references (e.g., similarity). This metric extends the concept and application of common systems of predetermined times (e.g., MTM) [160]. Based on the VDI-Richtlinie 2860 (assembly and handling) and relevant chapters of the DIN 8593-X (manufacturing processes), a representation of assembly primitives (as elemental operations) was developed in XML. Moreover, sequence weightings based on past incidents are modified to improve the search for optimal manufacturing and assembly sequences. Assembly instructions are derived via the resulting nonlinear assembly sequences.

4) Results: The instructions are presented to the worker via a multimodal interface including optical, acoustical, and touchas well as gesture-based information exchange. The variety of in- and output channels for human-machine-interaction enables optimal distribution to human perceptual resources. One key issue of the information presentation is the contact analog highlighting of relevant areas on the working area. In general, software-ergonomic principles are integrated into the dynamic assembly process. Moreover, several sensors are integrated in order to enable the context awareness of the system. Accordingly, distributed data and information acquisition from human workers, work places, and workpieces is established. The monitoring of the human worker includes gestures, expressed by hands and whole body movements. Additionally, fundamental results concerning attention allocation and action planning in applied scenarios are integrated. The vision-based scene observation utilizes these experimental results for switching the focus of attention to regions that are currently necessary.

Human cognition in production environments is investigated in controlled neurocognitive experiments in order to specify how the worker can be optimally supported. The experimental setup consists of a workbench equipped with a motion caption

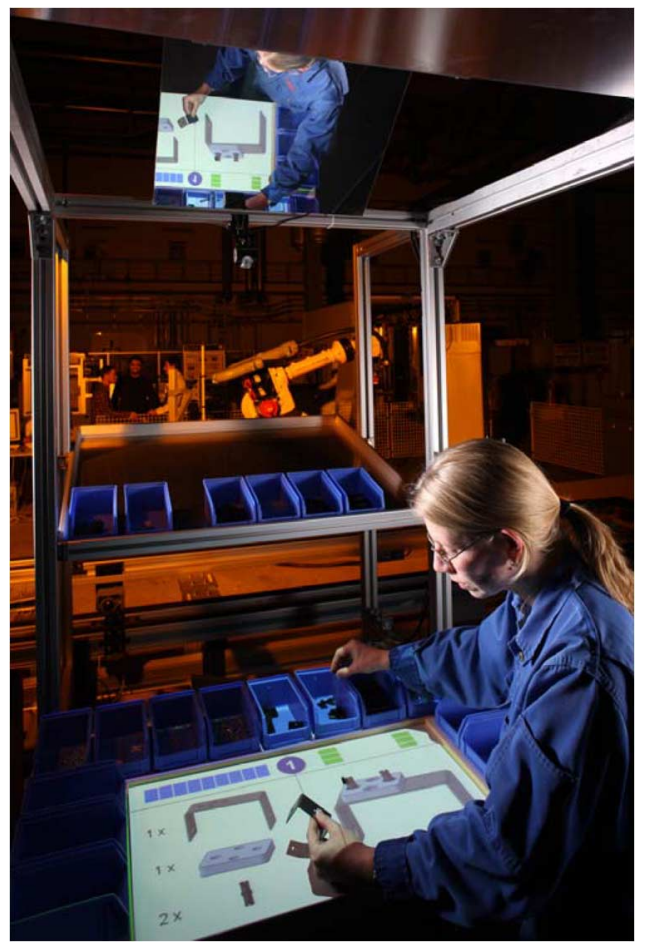

Fig. 28. Display of assembly instructions at the experimental workplace (CoTeSys/Kurt Fuchs).

system and an eye tracker in order to register hand, arm and eye movements during the whole assembly process (see Fig. 28). DV and firewire cameras are integrated for online and offline analyses of task performance. A beamer placed on top of the workbench together with a front-surface mirror enables the projection of assembly instructions directly on the work area.

During the experiments, task difficulty, task similarity, and task sequences are being varied. Results establish a database for human control processes to isolate cognitive processes involved in manual assembly and to derive relevant parameters for performance predictions. These behavioral measurement methods and parameters are integrated in the generation of worker-specific instructions.

Experiments are also used to evaluate both the interface and the mode of instruction presentation. Especially, methods for the guidance of the worker's attention were investigated. Experiments revealed an influence of task complexity and of communication modes on commissioning as well as on joining tasks. Movement onset time, peak velocity, and acceleration of the initial reaching and grasping movement were improved by contact analog highlighting in comparison to monitor presentations. Also, the projection of schematic box views in close proximity to the real boxes facilitated selection processes, because box positions could be compared without additional effort. Both conditions led to faster response execution of reaching and grasping movements. Thus, guiding visual attention and thereby reducing necessary attention shifts modulated performance times during commissioning [161]. In the joining task, various classes of assembly primitives differed substantially in assembly times. More complex operations such as orienting on two rotational axes versus orienting on all rotational axes took longer for single 
assembly parts, in accordance with traditional methods of predetermined times. The projection of task instructions close to the workpiece enabled faster completion of joining operations when more difficult spatial relationships had to be considered. For basic joining operations, no differences between projection and monitor presentation were found. From these results, one can conclude that complex assembly primitives benefit more from support by AR-based communication modes than easier ones. The experiments demonstrate an effect of task difficulty and attentional guidance on task performance [162], [163]. The observed interplay of mental processes (e.g., searching for assembly parts or for assembly positions) and action execution (e.g., grasping or joining) seems to be an indicator for task complexity and an important factor for the prediction of performance times.

In summary, the development of the proposed assistive system includes algorithms for dynamic work plan derivation. In the future, sensing technologies for real-time observation of humans are being developed. Further experiments will increase the understanding of human cognitive processes; their results will be implemented in control structures. This will enable the technical system to reason about the behavior of the human worker in single cases, to generalize knowledge of known events and to transfer it to new situations.

\section{SUMMARY}

In this paper, the demand for highly responsive and adaptive production systems is outlined, based on influence factors like volatile customer demands, short product lifecycles and the trend for individual and customized products.

While this turbulent environment has existed for manufacturing companies for several years, the approaches to deal with it have changed over time. In this paper, different production paradigms to ensure a (cost) efficient production are detailed and their historical development are described. Despite the achievements reached so far by these concepts, the silver bullet for highly responsive factories does not exist yet. In this paper, it is stated, that a key component for adaptive and reactive production systems are still human planners with their highly developed cognitive capabilities such as perceiving, planning, learning and acting. Therefore, a new paradigm of CTSs is introduced and its application to the factory domains is explained.

The approach of cognitive production systems may be applied to a wide range of problems and challenges in the factory domain. In this paper, the areas of production planning and control, automated processes and operations, as well as hybrid and manual processes and operations are introduced. Concrete topics in each of these three areas are outlined, including the respective state-of-the-art. In addition to the known concepts and approaches, for each problem, a possible solution using the approach of CTSs is introduced. The specific problems addressed, cover the area of production planning and control, autonomous machining and fixture planning, the automated programming of industrial robots, the human-robot-cooperation, knowledgebased quality assurance and process control, and worker guidance systems for manual assembly.
Applying the proclaimed paradigm of the Cognitive Factory will help to further advance the adaptivity, responsibility and changeability of production systems.

\section{ACKNOWLEDGMENT}

The authors thank Wolfgang Roesel for the coordination of this paper and Alexander Bannat for his technical support.

\section{REFERENCES}

[1] M. Mehrabi, A. Ulsoy, K. Y., and P. Heytler, "Trends and perspectives in flexible manufacturing systems," J. Intell. Manuf., vol. 13, no. 2, pp. 135-146, 2002.

[2] M. Zaeh and M. Ostgathe, "A multi-agent-supported, product-based production control," in Proc. IEEE Int. Conf. Control Automat. (ICCA'09), Christchurch, New Zealand, 2009.

[3] M. F. Zaeh, H. Rudolf, and N. Moeller, "Agile process planning considering the continuous reconfiguration of factories," in Proc. 3rd Int. Conf. Reconfigurable Manufacturing, CIRP10, Ann Arbor, MI, May 12, 2005.

[4] M. F. Zaeh, M. Beetz, K. Shea, G. Reinhart, K. Bender, C. Lau, M. Ostgathe, W. Vogl, M. Wiesbeck, M. Engelhard, C. Ertelt, T. Ruehr, M. Friedrich, and S. Herle, "The cognitive factory," in Changeable and Reconfigurable Manufacturing Systems, ser. Springer Series in Advanced Manufacturing, H. A. ElMaraghy, Ed. London, U.K.: Springer, 2009, pp. 355-371.

[5] Y. Koren, "General RMS characteristics. Comparison with dedicated and flexible systems," in Reconfigurable Manufacturing Systems and Transformable Factories, A. I. Dashchenko, Ed. Berlin, Germany: Springer-Verlag, 2006, pp. 27-45.

[6] B. Scholz-Reiter and M. Freitag, "Autonomous processes in assembly systems," Ann. CIRP, vol. 56, no. 2, pp. 712-729, 2007.

[7] M. F. Zaeh, N. Mueller, and C. Rimpau, "A holistic framework for enhancing changeability of production systems," in Proc. 1st Int. Conf. Changeable, Agile, Reconfigurable and Virtual Production (CARV 2005), Munich, Germany, 2005, pp. 77-84.

[8] A. G. Higuera and A. C. Montalyo, "RFID-enhanced multi-agent based control for a machining system," Int. J. Flexible Manuf. Syst., vol. 19, no. 1, pp. 41-61, 2007.

[9] G. Schuh, S. Gottschalk, and T. Höhne, "High resolution production management," Ann. CIRP, vol. 56, no. 1, pp. 439-442, 2007.

[10] R. Qiu, "RFID-enabled automation in support of factory integration," Robot. Comput.-Integrat. Manuf., vol. 23, no. 6, pp. 677-683, 2007.

[11] M. F. Zaeh and M. Ostgathe, "Approach for a decentralised productoriented production control," in Proc. 5th Int. Conf. Digital Enterprise Technol. (DET 2008), Nantes, France, Oct. 22-24, 2008.

[12] H. Putzer and R. Onken, "COSA-A generic cognitive system architecture based on a cognitive model of human behavior," Cognition, Technol. Work, vol. 5, no. 2, pp. 140-151, 2003.

[13] L. Monostori, J. Vünza, and S. R. T. Kumara, "Agent-based systems for manufacturing," Ann. CIRP, vol. 55, no. 2, pp. 697-719, 2006.

[14] F. Jovane, Y. Koren, and C. R. Boer, "Present and future of flexible automation: Towards new paradigms," Ann. CIRP, vol. 52, no. 2, pp. 543-560, 2003.

[15] Y. Koren, U. Heissel, F. Jovane, T. Moriwaki, G. Pritschow, A. G. Ulsoy, and H. van Brussel, "Reconfigurable manufacturing systems," Ann. CIRP, vol. 48, no. 2, pp. 527-540, 1999.

[16] M. R. Liu, Q. L. Zhang, L. M. Ni, and M. M. Tseng, "RFID-based distributed control system for mass customization manufacturing," in Lecture Notes in Computer Sciences. Berlin, Germany: Springer-Verlag, 2004, pp. 1039-1049.

[17] K. Ueda, J. Vaario, and K. Ohkura, "Modeling of biological manufacturing systems for dynamic reconfiguration," Ann. CIRP, vol. 46, no. 1, pp. 343-346, 1997.

[18] K. Ueda, I. Hatono, N. Fujii, and J. Vaario, "Line-less production system using self-organization: A case study for BMS," Ann. CIRP, vol. 50, no. 1, pp. 319-322, 2001.

[19] K. Ueda, T. Kito, and N. Fujii, "Modeling biological manufacturing systems with bounded-rational agents," Ann. CIRP, vol. 55, no. 1, pp. 469-472, 2006.

[20] K. Ueda, I. Hatono, N. Fujii, and J. Vaario, "Reinforcement learning approaches to biological manufacturing systems," Ann. CIRP, vol. 49, no. 1, pp. 343-346, 2000.

[21] P. Valckenaers and H. Van Brussel, "Holonic manufacturing execution systems," Ann. CIRP, vol. 54, no. 1, pp. 427-430, 2005. 
[22] M. Caridi and S. Cavalieri, "Multi-agent systems in production planning and control: An overview," Int. J. Prod. Econ., vol. 68, no. 1, pp. 29-42, 2000.

[23] M. Woolridge and N. R. Jennings, "Intelligent agents: Theory and practice," Knowl. Eng. Rev., vol. 10, no. 2, pp. 115-152, 1995.

[24] D. C. McFarlane, S. Sarma, J. L. Chirn, C. Y. Wong, and K. Ashton, "Auto ID systems and intelligent manufacturing control," Eng. Appl. Artif. Intell., vol. 16, no. 4, pp. 365-376, 2003.

[25] M. Zaeh, C. Lau, M. Wiesbeck, M. Ostgathe, and W. Vogl, "Towards the cognitive factory," in Int. Conf. Changeable, Agile, Reconfigurable and Virtual Production (CARV), Toronto, Canada, Jul. 2007.

[26] E. Hollnagel and P. C. Cacciabue, "Cognition, technology \& work: An introduction," Cognition, Technol. Work, vol. 1, no. 1, pp. 1-6, 1999.

[27] J. Rasmussen, A. M. Pejtersen, and L. P. Goodstein, Cognitive Systems Engineering, ser. Wiley series in system engineering, 3rd ed. New York: Wiley, 1994.

[28] M. J. Mataric, "Behavior-based robotics as tool for synthesis of artificial behavior and analysis of natural behavior," Trends in Cognitive Sciences, vol. 2, no. 3, pp. 82-87, 1998.

[29] J. M. Hoc, "Towards a cognitive approach to human-machine cooperation in dynamic situations," Int. J. Human-Computer Studies, vol. 54, no. 4, pp. 509-540, 2001.

[30] V. L. Shalin, "The roles of humans and computers in distributed planning for dynamic domains," Cognition, Technol. Work, vol. 7, no. 3, pp. 198-211, 2005.

[31] M. Beetz, M. Buss, and D. Wollherr, "“Cognitive technical systems'-What is the role of artificial intelligence?," in Proc. 30th Ann. German Conf. Advances in Artif. Intell., J. Hertzberg, M. Beetz, and R. Englert, Eds., Osnabrück, Germany, Sep. 10-13, 2007, vol. 4667, Lecture notes in computer science

[32] R. Brachman, "Systems that know what they're doing," IEEE Intell. Syst., vol. 17, no. 6, pp. 67-71, Nov./Dec. 2002.

[33] F. Stulp, M. Isik, and M. Beetz, "Implicit coordination in robotic teams using learned prediction models," in Proc. IEEE, Orlando, FL, May 15-19, 2006.

[34] F. Stulp and M. Beetz, "Optimized execution of action chains using learned performance models of abstract actions," in Proc. 19th Int. Joint Conf. Artif. Intell., L. P. Kaelbling, Ed., Edinburgh, Scotland, 2005.

[35] Y. Sallez, D. Trentesaux, T. Berger, and C. Tahon, "Product-based and resource-based heterarchical approaches for dynamic FMS scheduling," Comput. Ind. Eng., vol. 46, no. 4, pp. 611-623, 2004.

[36] M. Koike, R. Tsumaya, E. Matsui, H. Morinaga, H. Wakamatsu, and E. Arai, "Production scheduling system with dynamic lot size in case of considering set-up time," in Proc. 41st CIRP Conf. Manuf. Syst. Technol. New Frontier, Tokyo, Japan, 2008.

[37] G. Q. Huang, Q. L. Zhang, and P. Y. Jiang, "RFID-based wireless manufacturing for walking-worker assembly islands with fixed-position layouts," Robot. Comput.-Integrat. Manuf., vol. 23, no. 4, pp. 469-477, 2007.

[38] M. Zaeh, M. Ostgathe, C. Lau, and M. Wiesbeck, "Adaptive, productbased control of production processes," in Proc. 3rd Int. Conf. Changeable, Agile, Reconfigurable and Virtual Production (CARV 2009), Munich, Germany, 2009.

[39] K. Finkenzeller and R. Waddington, RFID Handbook: Fundamentals and Applications in Contactless Smart Cards and Identification, 2nd ed. Chichester: Wiley, 2006. [Online]. Available: http://www.loc.gov/catdir/description/wiley039/2002192439.html/

[40] W. E. Lulay and G. Reinhart, "Hierarchical simulation models improve production-accompanying-simulation," in Proc. 3rd Eur. Simulation Congr. (EuroSIM 98), Helsinki, Finnland, 1998, pp. 450-457.

[41] W. D. Kelton and A. Law, Simulation Modeling and Analysis. New York: McGraw-Hill, 1991.

[42] S. Mirdamadi, F. Fontanili, and L. Dupont, "Discrete event simulation-based real-time shop floor control," in Proc. 21st Eur. Conf. Modelling and Simulation (ECMS 2007), Prague, Czech Republic, 2007, pp. $235-240$.

[43] G. Andersson and G. Olsson, "A simulation based decision support approach for operationl capacity planning in a customer order driven assembly line," in Proc. 1998 Winter Simulation Conf., Washington, DC, 1998, pp. 935-941.

[44] C. S. J. Milberg, "Automatic generation of simulation models for production planning and control," Ann. German Academic Society for Production Engineering, vol. 10, no. 1, pp. 115-118, 2003.

[45] G. Weigert, S. Horn, T. Jähnig, and S. Werner, "Automated creation of DES models in an industrial environment," in Proc. 16th Int. Conf. Flexible Autom. Intell. Manuf. (FAIM 06), Limerick, Ireland, 2006, pp. 311-318.
[46] A. Thomas and P. Charpentier, "Reducing simulation models for scheduling manufacturing facilities," Eur. J. Oper. Res., vol. 161, pp. $111-125,2005$.

[47] G. Weigert, S. Werner, and W. Sauer, "Adaptive simulation systems for reducing uncertainty of predictions," in Proc. 9th Int. Conf. Flexible Autom. Intell. Manuf., Tilburg, The Netherlands, 1999, pp. 691-701.

[48] S. Werner, S. Horn, G. Weigert, and T. Jähnig, "Simulation based scheduling system in a semiconductor backend facility," in Proc. 2006 Winter Simulation Conf., Monterey, CA, 2006, pp. 1741-1748.

[49] G. Reinhart and T. Gyger, "Identification of implicit strategies in production control," in Proc. IEEE Int. Conf. Ind. Eng. and Eng. Manage. (IEEM 2008), Singapore, 2008, pp. 302-306.

[50] X. Li and S. Olafsson, "Discovering dispatching rules using data mining," J. Scheduling, vol. 8, pp. 515-527, 2005.

[51] J. A. Harding, M. Shahbaz, Srinivas, and A. Kusiak, "Data mining in manufacturing: A review," J. Manuf. Sci. Eng., vol. 128, pp. 969-976, 2006.

[52] EN 62264-1, Enterprise-Control System Integration-Part 1: Models and Terminology (IEC 62264-1:2003). 2008.

[53] C. Brecher, K. Fayzullin, and F. Possel-Dölken, "Intelligent operations control: Architecture for seamless integration of scheduling and execution," Prod. Eng., vol. 2, no. 3, pp. 293-301, 2008.

[54] H. Mosemann and F. Wahl, "Automatic decomposition of planned assembly sequences into skill primitives," IEEE Trans. Robot. Autom., vol. 17, no. 5, pp. 709-718, 2001.

[55] B. Finkemeyer, T. Krueger, and F. M. Wahl, "Executing assembly tasks specified by manipulation primitive nets," Advanced Robotics, vol. 19, no. 5, pp. 591-611, 2005.

[56] H. Van Brussel, "Planning and scheduling of assembly systems," Ann. CIRP, vol. 29, no. 2, pp. 637-644, 1990.

[57] S. Bussmann and K. Schild, "Self-organizing manufacturing control An industrial application of agent technology," in Proc. 4th Int. Conf. Multi-Agent Syst., Jul. 2000, pp. 87-94.

[58] W. Eversheim, F. Klocke, F. Hagemann, A. Kopner, and N. Michalas, "Knowledge management concept for production planning," Int. J. Prod. Eng. Comput., vol. 4, no. 5, pp. 19-24, 2002.

[59] G. Reinhart, R. Diesch, and M. Koch, "Knowledge-based information processing in manufacturing cells-The present and the future,", pp. 109-123, 1996

[60] F. Röhrdanz, H. Mosemann, and F. Wahl, "Highlap: A high level system for generating, representing, and evaluating assembly sequences," Int. J. Artif. Intell. Tools, vol. 6, no. 2, pp. 149-163, 1997.

[61] L. Gou, T. Hasegawa, P. B. Luh, S. Tamura, and J. M. Oblak, "Holonic planning and scheduling for a robotic assembly testbed," in Proc. 4th Int. Conf. Comput. Integr. Manuf. Autom. Technol., 1994, pp. 142-149.

[62] Industrial Automation Systems and Integration-Product Data Representation and Exchange-Part 214: Application Protocol: Core Data for Automotive Mechanical Design Processes, ISO/DIS 10303-214, 2003.

[63] G. Reinhart, T. Gyger, and C. Lau, "Modelling dynamic workflows in production control," in Proc. Conf. Changeable, Agile, Reconfigurable and Virtual Production (CARV 2007), Toronto, Canada, Jul. 2007, pp. 972-981.

[64] VDI guideline 2860, "Assembly and handling; Handling functions, handling units; terminology, definitions and symbols," 1990.

[65] J. Corney, C. Hayes, V. Sundararajan, and P. K. Wright, "The CAD/CAM interface: A 25-year retrospective," J. Comput. Inform. Sci. Eng., vol. 5, no. 3, pp. 188-197, 2005.

[66] H.-P. Wiendahl, H. A. ElMaraghy, P. Nyhuis, M. F. Zäh, H.-H. Wiendahl, N. Duffie, and M. Kolakowski, "Changeable manufacturingClassification, design and operation," Ann. CIRP, vol. 56, no. 2, 2007.

[67] W. D. Engelke, How to Integrate CAD/CAM Systems, ser. Mechanical Engineering. New York: Marcel Dekker, 1987.

[68] C. Ertelt, T. Rühr, D. Pangercic, K. Shea, and M. Beetz, "Integration of perception, global planning and local planning in the manufacturing domain," in Proc. 14th IEEE Int. Conf. Emerging Technol. Factory Autom., Mallorca, Spain, 2009

[69] S. Gupta, D. Nau, and W. Regli, "IMACS: A case study in real-world planning," IEEE Intell. Syst. Their Appl., vol. 13, no. 3, pp. 49-60, May/Jun. 1998.

[70] S. H. Ahn, V. Sundararajan, C. Smith, B. Kannan, R. D'Souza, G. Sun, A. Mohole, P. K. Wright, J. Kim, S. McMains, J. Smith, and C. H. Sequin, "CyberCut: An internet-based CAD/CAM system," J. Comput. Inform. Sci. Eng., vol. 1, no. 1, pp. 52-59, 2001.

[71] J. J. Shah, D. Anderson, Y. S. Kim, and S. Joshi, "A discourse on geometric feature recognition from CAD models," J. Comput. Inform. Sci. Eng., vol. 1, no. 1, pp. 41-51, 2001. 
[72] J. J. Shah and M. Mäntylä, Parametric and Feature-Based CAD/CAM: Concepts, Techniques, and Applications. New York: Wiley, 1995.

[73] R. T. Gruber, "A translation approach to portable ontology specifications," Knowle. Acquisition, vol. 5, no. 2, pp. 199-220, 1993.

[74] N. Guarino and P. Giaretta, "Ontologies and knowledge bases," in Proceedings, Towards Very Large Knowledge Bases, N. Mars, Ed. Amsterdam, The Netherlands: IOS Press, 1995, pp. 25-32.

[75] X. W. Xu, H. Wang, J. Mao, S. T. Newman, T. R. Kramer, F. M. Proctor, and J. L. Michaloski, "STEP-compliant NC research: The search for intelligent CAD/CAPP/CAM/CNC integration," Int. J. Prod. Res., vol. 43, no. 17, pp. 3703-3743, 2005.

[76] X. Xu, L. Wang, and Y. Rong, "STEP-NC and function blocks for interoperable manufacturing," IEEE Trans. Autom. Sci. Eng., vol. 3, no. 3, pp. 297-308, Jul. 2006.

[77] J. Rosso, S. U. Roberto, R. Allen, and S. T. Newman, "Future issues for CAD/CAM and intelligent CNC manufacture," in Proc. 19th Int. Manuf. Conf.-IMC-19, North Ireland, UK, 2002, Queen's University Belfast.

[78] C. Ertelt and K. Shea, "An application of shape grammars to planning for CNC machining," in Proc. ASME 2009 Int. Design Engineering Tech. Conf. Comput. Inform. Eng. Conf., IDETC/CIE, San Diego, CA, 2009.

[79] G. Stiny, "Introduction to shape and shape grammars," Environment and Planning B: Planning and Design, vol. 7, no. 3, pp. 343-351, 1980.

[80] E. Ostrosi and M. Ferney, "Feature modeling using a grammar representation approach," Artif. Intell. Eng. Design, Anal. Manuf., AI EDAM, vol. 19, no. 4, pp. 245-259, 2005.

[81] M. Agarwal and J. Cagan, "On the use of shape grammars as expert systems for geometry-based engineering design," Artif. Intell. Eng. Design, Anal. Manuf., AI EDAM, vol. 14, no. 5, pp. 431-439, 2001.

[82] M. Agarwal and J. Cagan, "A blend of different tastes: The language of coffee makers," Environment and Planning B: Planning and Design, vol. 25, no. 2, pp. 205-226, 1998.

[83] M. Pugliese and J. Cagan, "Capturing a rebel: Modeling the HarleyDavidson brand through a motorcycle shape grammar," Res. Eng. Design, vol. 13, no. 3, pp. 139-156, 2002.

[84] A. C. Starling and K. Shea, "A parallel grammar for simulation-driven mechanical design synthesis," in Proc. ASME Int. Design Eng. Tech. Conf. Comput. Inform. Eng., IDETC/CIE, Long Beach, CA, 2005.

[85] L. Sass, K. Shea, and M. Powell, "Design production: Constructing free-form designs with rapid prototyping," in ECAADE, Lisbon, Portugal, 2005.

[86] K. N. Brown, C. A. McMahon, and J. H. Sims Williams, "Features, aka the semantics of a formal language of manufacturing," Res. Eng. Design, vol. 7, no. 3, pp. 151-172, 1995.

[87] A. Shirur, J. J. Shah, and K. Hirode, "Machining algebra for mapping volumes to machining operations for developing extensible generative CAPP," J. Manuf. Syst., vol. 17, no. 3, pp. 167-182, 1998.

[88] C. Ertelt and K. Shea, "Generative design and CNC fabrication using shape grammars," in Proc. ASME 2008 Int. Design Eng. Tech. Conf. Comput. Inform. Eng., IDETC/CIE, Brooklyn, NY, 2008.

[89] M. Ghallab, E. Nationale, C. A. C. K. Isi, K. Golden, S. Penberthy, D. E. Smith, Y. Sun, D. Weld, and C. D. Mcdermott, PDDL-The planning domain definition language, version 1.2, Tech. Rep., 1998.

[90] J. Hoffmann, Dissertation: Utilizing problem structure in planning: a local search approach Albert-Ludwigs-Universität Freiburg, Tech. Rep, 2003,

[91] Open CASCADE S.A.S., "Open CASCADE technology, 3D modeling \& numerical simulation," 2008

[92] R. Hooke and T. A. Jeeves, "Direct search-Solution of numerical and statistical problems," J. ACM (JACM), vol. 8, no. 2, pp. 212-229, 1961.

[93] S. Kirkpatrick, "Optimization by simulated annealing: Quantitative studies," J. Statist. Phys., vol. 34, no. 5, pp. 975-986, 1984.

[94] K. Shea, M. Engelhard, C. Ertelt, and F. Hoisl, "A framework for a cognitive design-to-fabrication system," in Proc. 7th Int. Symp. Tools and Methods of Competitive Engineering (TMCE 2008), Ismir, Turkey, 2008.

[95] Z. Bi and W. Zhang, "Flexible fixture design and automation: Review, issues and future directions," Int. J. Prod. Res., vol. 39, no. 13, pp. 2867-2894, 2001

[96] A. Felix, S. Melkote, and Y. Matsumoto, "Modeling of the holding force in an electromagnetic chuck," J. Manuf. Sci. Eng., vol. 122, p. 7, 2000.

[97] Y. Rong, R. Tao, and X. Tang, "Flexible fixturing with phase-change materials. Part 1. Experimental study on magnetorheological fluids," Int. J. Advanced Manuf. Technol., vol. 16, no. 11, pp. 822-829, 2000.
[98] S. Kamarthi, N. Bhole, and A. Zeid, "Investigating the design and development of truly agile flexible fixtures based on electrorheological fluids," Int. J. Rapid Manuf., vol. 1, no. 1, pp. 99-110, 2009.

[99] R. Brown and R. Brost, "A 3-D modular gripper design tool," IEEE Trans. Robot. Autom., vol. 15, no. 1, pp. 174-186, 1999.

[100] S. Lu, J. Chu, and H. Jang, "Development of a novel coordinate transposing fixture system," Int. J. Advanced Manuf. Technol., vol. 13, no. 5, pp. 350-358, 1997.

[101] J. Hurtado and S. Melkote, "A model for synthesis of the fixturing configuration in pin-array type flexible machining fixtures," Int. J. Machine Tools and Manuf., vol. 42, no. 7, pp. 837-849, 2002.

[102] A. Kumar, J. Fuh, and T. Kow, "An automated design and assembly of interference-free modular fixture setup," Comput.-Aided Design, vol. 32 , no. 10 , pp. 583-596, 2000.

[103] F. Ameri and J. D. Summers, "An ontology for representation of fixture design knowledge," Comput.-Aided Design Appl., vol. 5, no. 5, pp. 601-611, 2008.

[104] S. Pehlivan and J. D. Summers, "A review of computer-aided fixture design with respect to information support requirements," Int. J. Prod. Res., vol. 46, no. 4, pp. 929-947, 2008.

[105] J. Cecil, "Computer-aided fixture design-a review and future trends," Int. J. Advanced Manuf. Technol., vol. 18, no. 11, pp. 790-793, 2001.

[106] C. Ertelt, T. Gmeiner, and K. Shea, "A flexible fixture and reconfiguration process for the cognitive machine shop," in Proc. 3rd Int. Conf. Changeable, Agile, Reconfigurable and Virtual Production (CARV 2009), Munich, Germany, 2009.

[107] G. Liu, J. Xu, X. Wang, and Z. Li, "On quality functions for grasp synthesis, fixture planning, and coordinated manipulation," IEEE Trans. Autom. Sci. Eng., vol. 1, no. 2, pp. 146-162, Jan. 2004.

[108] X. Zhu and D. Han, "Optimality criteria for fixture layout design: A comparative study," IEEE Trans. Autom. Sci. Eng., vol. 6, no. 4, p. 12, Oct. 2009.

[109] L. Yadong and G. Peihua, "Free-form surface inspection techniques state of the art review," Comput.-Aided Design, vol. 36/13, pp. 1395-1417, 2004.

[110] F. Prieto, T. Redarce, P. Boulanger, and R. Lepage, "CAD-based range sensor placement for optimum 3D data acquisitio," in Proc. 2nd Int. Conf. 3D Digital Imaging and Modeling (3DIM '99), Ottawa, Canada, Oct. 4-8, 1999, pp. 128-137.

[111] S. Son, H. Park, and K.-H. Lee, "Automated laser scanning system for reverse engineering and inspection," Int. J. Machine Tools and Manuf., vol. 42/8, pp. 889-897, 2002.

[112] H. Y. X. ElMaraghy, "Computer-aided planning of laser scanning of complex geometries," Ann. CIRP, vol. 52/1, pp. 411-414, 2003.

[113] P. Fernandez, B. J. Alvarez, J. C. Rico, D. Blanco, and G. Valino, "Constraints evaluation and working methodology for laser scanning of free-form surfaces," in Proc. IEEE Conf. Emerging Technol. Factory Autom., 2006, pp. 523-530.

[114] A. Bernard and M. Veron, "Analysis and validation of 3D laser sensor scanning process," in Ann. CIRP 1999, 1999, vol. 48/1, pp. 111-114.

[115] W. R. Scott, G. Roth, and J.-F. Rivest, "View planning for automated three-dimensional object," ACM Comput. Surveys, vol. 35/1, pp. 64-96, 2003.

[116] F. Bertagnolli, Robotergestützte automatische Digitalisierung von Werkstückgeometrien mittels optischer Streifenprojektion: Univ., Diss.-Karlsruhe, 2006, ser. Messtechnik und Sensorik. Aachen, Germany: Shaker, 2006.

[117] S. Larsson, An Industrial Robot as Carrier of a Laser Profile Scanner: Motion Control, Data Capturing and Path Planning: Diss., ser. Örebro studies in technology. Örebro: Örebro Universitet, 2008.

[118] M. Callieri, A. Fasano, G. Impoco, C. P. , and S. R. , "Roboscan: An automatic system for accurate and unattended 3D scanning," in Proc. 2nd IEEE Int. Symp. 3D Data Process., Visualization and Transmission, 2004, pp. 805-812.

[119] C. Shu and F. Xi, "Model-based scanning path generation for inspection," in Proc. 2nd Int. Conf. 3-D Digital Imaging and Modeling, 1999, pp. $118-124$

[120] C. Roessl, L. Kobbelt, and H.-P. Seidel, "Extraction of feature lines on triangulated surfaces using morphological operators," in Proc. AAAI Spring Symp. Series-Smart Graphics, Stanford, 2000, pp. 71-75.

[121] I. Guskov, W. Sweldens, and P. Schroeder, "Multiresolution signal processing for meshes," in Proc. 26th Ann. Conf. Comput. Graphics and Interactive Tech., Los Angeles, CA, 1999, pp. 325-334.

[122] E. Larsen, S. Gottschalk, M. C. Lin, and D. Manocha, "Fast proximity queries with swept sphere volumes," in Proc. Int. Conf. Robot. Autom., San Francisco, CA, 2000, pp. 3719-3726. 
[123] F. Zacharias, C. Borst, and G. Hirzinger, "Capturing robot workspace structure: Representing robot capabilities," in Proc. IEEE Int. Conf. Intell. Robot. Syst., San Diego, CA, 2007, pp. 3229-3236.

[124] C. Schwarzer, M. Saha, and J.-C. Latombe, "Adaptive dynamic collision checking for single and multiple articulated robots in complex environments," IEEE Trans. Robotics, vol. 21/3, pp. 338-353, 2005.

[125] N. Sebanz, H. Bekkering, and G. Knoblich, "Joint action: Bodies and minds moving together," Trends in Cognitive Sciences, vol. 10, no. 2, pp. 70-76, Feb. 2006.

[126] A. A. Argyros and M. I. A. Lourakis, "Real-time tracking of multiple skin-colored objects with a possibly moving camera," in Proc. ECCV (3), 2004, pp. 368-379.

[127] S. Reifinger, F. Wallhoff, M. Ablaßmeier, T. Poitschke, and G. Rigoll, "Static and dynamic hand-gesture recognition for augmented reality applications," in Proc. Int. Conf. Human-Comput. Interaction, C. Stephanidis, Ed., Beijing, China, Jul. 2007.

[128] N. J. Emery, "The eyes have it: The neuroethology, function and evolution of social gaze," Neuroscience and Biobehavioral Reviews pp. 581-604, Aug. 2000. [Online]. Available: http://www.ingentaconnect. com/content/els/01497634/2000/00000024/00000006/art00025

[129] G. Panin and A. Knoll, "Mutual information-based 3D object tracking," Int. J. Comput. Vision (IJCV), 2007.

[130] C. Lenz, S. Nair, M. Rickert, A. Knoll, W. Rösel, J. Gast, and F. Wallhoff, "Joint-action for humans and industrial robots for assembly tasks," in Proc. IEEE Int. Symp. Robot and Human Interactive Commun., 2008, pp. 130-135.

[131] S. Bardins, T. Poitschke, and S. Kohlbecher, "Gaze-based interaction in various environments," in Proc. 1st ACM Int. Workshop on Vision Networks for Behavior Analysis, VNBA 2008, Vancouver, Canada, October 31, 2008.

[132] M. Zaeh and W. Roesel, "Safety Aspects in a Human-Robot Interaction Scenario: A Human Worker is Co-Operating With an Industrial Robot," FIRA. Incheon, Korea, 16. bis 18, Aug. 2009.

[133] A. Bannat, J. Gast, T. Rehrl, W. Rösel, G. Rigoll, and F. Wallhoff, "A multimodal human-robot-interaction scenario: Working together with an industrial robot," in Proc. Int. Conf. on Human-Comput. Interaction HCI 2009, J. Jacko, Ed., San Diego, CA, 2009, vol. 5611, LNCS, pp. 303-311, ISBN 978-3-642-02576-1, Springer, 19.-24.07. 2009, Human-Computer Interaction. Novel Interaction Methods and Techniques.

[134] P. Vadakkepat, P. Lim, L. De Silva, L. Jing, and L. L. Ling, "Multimodal approach to human-face detection and tracking," IEEE Trans. Ind. Electron., vol. 55, no. 3, pp. 1385-1393, March 2008.

[135] I. Stork genannt Wersborg, F. Borgwardt, and K. Diepold, "Real-time cognitive technical systems, a learning material processing system, social and future aspects," IEEE/ARSO, Advanced Robotics and Its Social Impacts, Nov. 2009.

[136] J. Mueller-Borhanian, C. Deininger, F. Dausinger, and H. Hugel, "Spatially resolved online monitoring during laser beam welding of steel and aluminum," in Proc. 23rd ICALEO, 2004. [Online]. Available: http://www.fgsw.de/iness/aktuelles/icaleo_2004.pdf

[137] S. Kaierle, "Process monitoring and control of laser beam welding," Laser Technik J., vol. 5, no. 3, pp. 41-43, 2008.

[138] F. Abt, L. Nicolosi, D. Carl, A. Blug, M. Geese, F. Dausinger, C. Deininger, H. Höfler, and R. Tetzlaff, "Closed loop control of laser welding processes with cellular neural network (CNN) cameras," in Proc. 27th Int. Congr. Applicat. Laser \& Electro Optics, 2008.

[139] A. Bollig, D. Abel, C. Kratzsch, and S. Kaierle, "Identification and predictive control of laser beam welding using neural networks," in Proc. Eur. Control Conf., ECC, 2003. [Online]. Available: http://www. scientificcommons.org/20346260

[140] H. Luo, H. Zeng, L. Hu, X. Hu, and Z. Zhou, "Application of artificial neural network in laser welding defect diagnosis," J. Mater. Process. Technolo., vol. 170, no. 1-2, pp. 403-411, 2005.

[141] M. Jaeger, C. Knoll, and F. Hamprecht, "Weakly supervised learning of a classifier for unusual event detection," IEEE Trans. Image Process., vol. 17, no. 9, pp. 1700-1708, 2008.

[142] I. Stork genannt Wersborg, K. Schorp, T. Bautze, and K. Diepold, "Multiple sensors and artificial neural networks in a cognitive technical system for laser welding," in Proc. IEEE/ISSNIP, Intelligent Sensors, Sensor Networks and Info., Dec. 2009, accepted for publication.

[143] N. Sonnde, "A Comparison of Cognitive Approaches for Quality Control of Laser Beam Cutting," Master's thesis, TU München, , 2008

[144] S. Hilsenbeck, "High dynamic range imaging for laser material processing," Master's thesis, Technische Universität München, Munich, Germany, Aug. 2009.
[145] I. Stork genannt Wersborg, T. Bautze, F. Born, and K. Diepold, “A cognitive approach for a robotic welding system learning how to weld from acoustic data," in Proc. IEEE/CIRA Int. Symp. Comput. Intell. Robot. Autom., 2009.

[146] T. Bautze, K. Diepold, and T. Kaiser, "A cognitive approach to monitor and control focal shifts in laser beam welding applications," in Proc. IEEE/ICIS Int. Conf. Intell. Comput. Intell. Syst., 2009, pp. 895-899.

[147] K. Schorp, "Cognitively controlled laser beam welding and artificial neural networks," Master's thesis, Technische Universität München, Munich, Germany, Mar. 2009.

[148] T. Bautze, "Fingerprinting laser material processes in a technical cog nitive environment," Master's thesis, Universitat Karlsruhe (TH), Karlsruhe, Germany, 2009.

[149] T. Bautze, T. Kaiser, and S. Huber, "Focal shift monitoring and cognitive closed-loop control by analysis of co-axial video data," in Proc. ICALEO Conf., 2009, pp. 849-855.

[150] G. Schroth, I. Stork genannt Wersborg, and K. Diepold, "A cognitive system for autonomous robotic welding," in Proc. IEEE/RSJ IROS Intell. Robot. Syst., 2009.

[151] W. H. ElMaraghy and R. J. Urbanic, "Assessment of manufacturing operational complexity," Ann. CIRP, vol. 53, no. 1, pp. 401-406, 2004.

[152] P. L. Jensen and A. Leo, "Human factors in the management of production," Ann. CIRP, vol. 55, no. 1, 2006.

[153] M. Zaeh and M. Prasch, "Systematic workplace and assembly redesign for aging workforces," Prod. Eng., vol. 1, no. 1, pp. 57-64, 2007.

[154] J. E. Ilmarinen, "Aging workers," Occupational and Environmental Medicine, vol. 58, no. 8, pp. 546-552, 2001.

[155] M. F. Zaeh, M. Wiesbeck, F. Wallhoff, S. Stork, F. Engstler, A. Bannat, A. Schuboe, and F. Friesdorf, "Kognitive assistenzsysteme in der manuellen montage," wt Werkstattstechnik Online, vol. 97, no. 9, pp. $644-650,2007$.

[156] G. Reinhart and C. Patron, "Integrating augmented reality in the assembly domain-Fundamentals, benefits and applications," Ann. CIRP, vol. 52, no. 1, pp. 5-8, 2003.

[157] L. L. Dopping-Hepenstal, "Head-up displays: The integrity of flight information," Proceed. IEEE Commun., Radar and Signal Process., vol. 128, no. 7, pp. 440-442, 1981.

[158] M. Yeh and C. D. Wickens, "Attention and trust biases in the design of augmented reality displays,", 2000.

[159] M. Wiesbeck and M. F. Zaeh, "A model for adaptively generating assembly instructions using state-based graphs," in Proc. 41st CIRP Conf. Manuf. Syst., M. Mitsuishi, K. Ueda, and F. Kimura, Eds., Tokyo, Japan, 2008, pp. 195-198.

[160] M. F. Zaeh, M. Wiesbeck, S. Stork, and A. Schuboe, "Factors for a task-induced complexity measure for manual assembly operations," in Proc. 3rd Int. Conf. Changeable, Agile, Reconfigurable and Virtual Production (CARV 2009), M. F. Zaeh, Ed., München, Germany, 2009, pp. 831-845.

[161] S. Stork, C. Stoessel, and A. Schuboe, "The influence of instruction mode on reaching movements during manual assembly," in $\mathrm{HCI}$ and Usability for Education and Work, ser. 4th Symposium of the Workgroup Human-Computer Interaction and Usability Engineering of the Austrian Computer Society, A. Holzinger, Ed. Heidelberg, Germany: Springer-Verlag, 2008, vol. 5298, LNCS, pp. 161-172.

[162] C. Stoessel, C. Wiesbeck, S. Stork, M. F. Zaeh, and A. Schuboe, "Towards optimal worker assistance: Investigating cognitive processes in manual assembly," in Proc. 41st CIRP Conf. Manuf. Syst., 2008, pp. 245-250.

[163] S. Stork, C. Stoessel, and A. Schuboe, "Optimizing human-machine interaction in manual assembly," in Proc. 17th IEEE Int. Symp. Robot and Human interactive Commun., RO-MAN 2008, 2008.

Alexander Bannat, photograph and biography not available at the time of publication.

Thibault Bautze, photograph and biography not available at the time of publication.

Michael Beetz, photograph and biography not available at the time of publication. 
Juergen Blume, photograph and biography not available at the time of publication.

Klaus Diepold, photograph and biography not available at the time of publication.

Christoph Ertelt, photograph and biography not available at the time of publication.

Florian Geiger, photograph and biography not available at the time of publication.

Thomas Gmeiner, photograph and biography not available at the time of publication.

Tobias Gyger, photograph and biography not available at the time of publication.

Alois Knoll, photograph and biography not available at the time of publication.

Christian Lau, photograph and biography not available at the time of publication.

Claus Lenz, photograph and biography not available at the time of publication.

Martin Ostgathe, photograph and biography not available at the time of publication.

Gunther Reinhart, photograph and biography not available at the time of publication.

Wolfgang Roesel, photograph and biography not available at the time of publication.
Thomas Ruehr, photograph and biography not available at the time of publication.

Anna Schuboe, photograph and biography not available at the time of publication.

Kristina Shea, photograph and biography not available at the time of publication.

Ingo Stork genannt Wersborg, photograph and biography not available at the time of publication.

Sonja Stork, photograph and biography not available at the time of publication.

William Tekouo, photograph and biography not available at the time of publication.

Frank Wallhoff, photograph and biography not available at the time of publication.

Mathey Wiesbeck, photograph and biography not available at the time of publication.

Michael F. Zaeh received the Doctorate degree from the Institute of Machine Tools and Industrial Management, Technical University of Munich, Munich, Germany, in 1993, with a thesis about a dynamic process model for circular sawing

He was born in Coburg/Bavaria/Germany, in 1963. He started his career in 1990, assuming a position as Graduate Research Assistant at the Institute of Machine Tools and Industrial Management, Technical University of Munich. In 1996, he joined Gleason-Pfauter Maschinenfabrik $\mathrm{GmbH}$, a builder of machine tools for manufacturing of gears, where he worked in the research laboratory and in the design department during the first year. From 1998 to 2002, he worked in an ERP-system-implementation project at four production sites and three sales agencies of the Gleason Corporation. During that time, he stayed in Rochester, NY, for two years. He worked also in South Korea, Great Britain, Brazil, and Japan. In 2002, he accepted the position of Full Professor for machine tools and manufacturing technology. Prof. Dr. Michael Zaeh is a member of acatech, the German Academy of Technical Science of the WGP (German Scientific Society for Production Technology), WLT (Scientific Society of Laser Technology), as well as of several other institutions. 